\title{
The Quest for Status and Endogenous Labor Supply: the Relative Wealth Framework
}

\author{
Walter H. FISHER* $\quad$ Franz X. $\mathrm{HOF}^{\dagger}$
}

November 2005

\begin{abstract}
This paper introduces the quest for status into the Ramsey model with endogenous labor supply. We focus our attention on relative wealth preferences. In contrast to relative consumption preferences, they allow for the possibility that agents work too little in the long run, while under both specifications the steady-state levels of consumption and the stock of physical capital exceed their socially optimal counterparts. The initial phase of transitional dynamics is unambiguously characterized by under-consumption and excessive work effort. The social optimum can be replicated by taxing capital income, where the optimal tax rate increases as physical capital accumulates.

JEL classification: D62, D91, E21.
\end{abstract}

Keywords: Status, relative consumption, relative wealth, endogenous labor supply.

* Institute for Advanced Studies, Department of Economics and Finance, Stumpergasse 56, A-1060 Vienna, Austria. Ph: +43-159991-253; Fax: -555; Email: fisher@ihs.ac.at.

${ }^{\dagger}$ Institute for Mathematical Methods in Economics, Research Unit Economics, Vienna University of Technology, Karlsplatz 13, A-1040 Vienna, Austria. Phone: +43-1-58801-17566, Fax: +43-1-58801-17599, Email: fxhof@pop.tuwien.ac.at.

We wish to thank Jürgen von Hagen, Oliver Landmann, Monika Merz, and Gerhard Schwödiauer for their very helpful comments on an earlier version of this paper. Fisher also thanks the Oesterreichische Nationalbank (OeNB) for its very generous financial support (Jubiläumsfondsprojekt Nr. 8701). 


\section{Introduction}

The goal of this paper is to investigate the influence of the quest for status in a Ramseytype model with endogenous labor supply and homogenous agents. In macroeconomics it has been the practice of most researchers to assume that status, modeled in terms of instantaneous preferences, depends either on relative consumption or on relative wealth. While our model includes both specifications of status, we will emphasize the implications of the latter, relative wealth. In our work, we will employ not only a general specification of preferences, but also a specific illustration that will allow us to conduct a very detailed analysis of the impact of status on the transitional dynamics.

The relative consumption approach has been used by researchers such as Boskin and Sheshinski (1978), Galí (1994), Persson (1995), Harbaugh (1996), Rauscher (1997), Grossmann (1998), Ljungqvist and Uhlig (2000), Fisher and Hof (2000a,b), Dupor and Liu (2003), Abel (2005), and Liu and Turnovsky (2005). In contrast, the relative wealth approach is employed in Corneo and Jeanne (1997, 2001a,b), Futagami and Shibata (1998), Hof and Wirl (2003), Fisher (2004), Van Long and Shimomura (2004a, b), and Fisher and Hof (2005). In the majority of this research the supply of labor is exogenously given. ${ }^{1}$ This turns out to be a restrictive assumption. In models with fixed employment that abstract from physical depreciation and technical progress, such as Rauscher (1997) and Fisher and Hof (2000a), the steady-state values of consumption and capital are independent of consumption externalities: only transitional dynamics is affected. Moreover, Fisher and Hof (2000a) have shown that in the fixed employment case there exist several quite general types of instantaneous utility functions in which the decentralized solution is efficient in spite of the existence of consumption externalities. If, on the other hand, work effort is endogenously determined, then i) not only is the dynamic behavior of the economy affected by a preference for status, but also the properties of its stationary equilibrium, and ii) the quest for status always gives rise to inefficient decentralized solutions. For these reasons we believe that it is interesting to consider the implications of status preferences

\footnotetext{
${ }^{1}$ There are a few exceptions in which labor supply is treated as endogenously determined. This research differs from our paper as follows: Persson (1995) considers heterogeneous agents, but neglects any intertemporal considerations and relative wealth. Fisher and Hof (2000b) restrict attention to consumption externalities. The framework in Dupor and Liu (2003) is static and ignores relative wealth. Liu and Turnovsky (2005) consider both exogenous and endogenous labor supply, but do not analyze relative wealth externalities.
} 
in the endogenous employment setting. Our extension allows us first to consider whether in the long run status-conscious people work "too much" or "too little", compared to the social optimum and whether the answer to this question depends on the way status is modelled. In addition, we examine the short- and medium-term deviation of the decentralized solution from its socially optimal counterpart. Furthermore, we analyze whether the inefficiencies resulting from status preferences can be removed by an appropriate tax policy.

To focus on the consequences of status preferences, we keep the rest of our economic framework as simple as possible, e.g., we employ a standard constant returns to scale production function and abstract from factors such as technological progress and physical depreciation. While our specification of instantaneous preferences accommodates both relative consumption and relative wealth, our contribution, as indicated above, centers on the short and long-run implications of the latter. Moreover, among the other goals of the paper is the comparison of the long-run properties of economies with relative wealth preferences to those in which status is a function of relative consumption. In certain respects, the effects of relative consumption and relative wealth externalities on the steady-state equilibrium of the decentralized economy are similar, e.g., both lead (identical) agents to consume "too much" and to accumulate an "excessive" stock of physical capital. In other respects, however, the two specifications can have very different long-run implications. For example, we demonstrate in our general preference specification that it is not necessarily the case that agents supply "too much" labor in the relative wealth framework. Indeed, we show in the first part of the paper that it is possible that agents work "too little" in this setting, a result that stands in contrast to the findings of recent researchers such as Fisher and Hof (2000b) and Liu and Turnovsky (2005), who prove, under general assumptions, that agents work "too hard" if social status depends on the level of average consumption. Another distinction between the two approaches is that the inefficiencies stemming from relative wealth approach are due to the fact that the effective rate of return of wealth exceeds the market rate, while in the relative consumption framework the private equilibrium is inefficient because the willingness to substitute consumption for leisure is too high.

Another focus of the paper is an analysis of the transitional dynamics of an economy characterized by relative wealth preferences. To do so, we adopt in the second half 
of the paper a simple parameterization of preferences in which work effort is additively separable from consumption and relative wealth and in which the intertemporal elasticity of substitution for consumption is constant in the symmetric equilibrium. A crucial advantage of this illustration is that it yields a unique, saddlepoint-stable steady state, properties that do not (necessarily) hold for the general formulation of preferences employed in the first part of the paper. A further advantage of this particular specification is that the socially optimal solution is obtained simply by setting the status parameter equal to zero in the corresponding solutions of the decentralized economy. Note, however, that the latter characteristic does not obtain generally and, in particular, does not hold in the relative consumption model of Harbaugh (1996) that is further analyzed by Fisher and Hof (2000a).

Using the illustration, we are able to derive the economy's linearized dynamics in state-control space, i.e., in terms of physical capital and, respectively, consumption and employment. Specifically, we employ this analytical framework to consider the effects of a higher degree of status preference on the economy's transitional dynamics. We demonstrate that in the initial phase of adjustment agents in the decentralized economy consume "too little" and work "too hard" compared to the values that would obtain in the socially optimal setting. We supplement the analytical results of this part of the paper with phase diagrams that describe the co-movements of physical capital and, in turn, consumption and work effort. The phase diagrams assist us in showing how the steady states and initial values for the control variables in the decentralized economies differ from their socially optimal counterparts. Another notable finding from this part of the paper includes the fact that for all admissible parameter values, an increase in the status parameter "slows down" the economy's speed of convergence along stable saddle path.

We continue our analysis of the specific parameterization of preferences by simulating numerically the adjustment paths of consumption and work effort for the linearized dynamics. This exercise allows us to display properties of the economy's intertemporal adjustment that are not completely revealed by the phase diagrams. Restricting attention to the case in which the initial value of capital is below its steady-state level, we show that the decentralized and socially optimal paths of consumption "cross", in the sense that initial under-consumption becomes medium-term and long-run over-consumption. Indeed, this is a necessary counterpart of the fact that status-conscious agents accumulate "too much" 
physical capital during the transition to steady state. Regarding the transitional dynamics of work effort, we show that its behavior in both the decentralized and the socially planned economy depends crucially on the difference between the parameter describing the (inverse) of the elasticity of intertemporal substitution of consumption and the share of capital in national income. For the case corresponding to an empirically plausible, i.e., "low" value of the elasticity of intertemporal substitution, the paths of decentralized and socially optimal employment "cross", since in this case agents work "too hard" initially, but subsequently "too little." Finally, we complete the analysis of the illustration by briefly treating the question of optimal taxation. Here, we prove that an optimal tax on capital income ensures that the decentralized economy reproduces the social optimum and show that the tax rate rises along with the physical capital stock.

The rest of the paper is organized as follows: section 2 describes the basic macroeconomic model and studies the social planner's problem. The first part of section 3 analyzes the decentralized framework for the general model of preferences and studies the deviation of its steady state from its socially optimal counterpart. The next part of section 3 uses the specific parameterization of preferences to study the saddle path adjustment of the solution of the linearized model. As indicated above, this includes the initial response of consumption and work effort along their respective saddle paths as well as the transitional adjustment of these variables compared to their socially optimal counterparts. The last part of section 3 briefly discusses the issue of optimal taxation of capital income. Section 4 contains some brief concluding remarks. The paper closes with an appendix that contains some mathematical results and proofs referred to in the main text.

\section{The Model and the Social Planner's Problem}

\subsection{Specification of the Model}

The economy is populated by a large number of identical, infinitely-lived individuals. For simplicity, we assume that the population size remains constant over time. As is usual in the Ramsey framework, we restrict attention to the case in which agents possess perfect foresight. The representative individual chooses the time paths of own consumption $c$ and own work effort (measured by hours worked) $l$ in order to maximize discounted intertemporal utility, which is given by $\int_{0}^{\infty} e^{-\rho t} u(c, l, z) d t$, where $\rho$ is the constant rate 
of time preference, $u$ denotes the instantaneous utility function, and $z$ denotes a variable that determines the agent's relative position, or status, in society. In our model status is either determined by relative consumption, i.e., $z \equiv c / C$, where $C$ denotes the average (or per capita) consumption in the economy, or by relative wealth, i.e., $z \equiv a / A$, where $a$ is the agent's own nonhuman wealth, while $A$ denotes the average wealth in the economy. As indicated above, we focus in this paper on the latter case in which the status variable is relative wealth. These specifications imply that in both approaches leisure is assumed to be a non-positional good. ${ }^{2}$

We further assume that $u$ possesses continuous first-order and second-order partial derivatives that have the following usual properties:

$$
\begin{gathered}
u_{c}>0, u_{c c}<0, u_{l}<0, u_{l l}<0, u_{z}>0, u_{z z}<0, u_{c c} u_{l l}-u_{c l}^{2}>0, \\
u_{l c} u_{c}-u_{l} u_{c c}<0, \quad u_{l l} u_{c}-u_{l} u_{c l}<0 .
\end{gathered}
$$

According to (1), the representative individual derives positive and diminishing marginal utility from both own consumption and her relative position in society as measured by the variable $z$, in addition to positive and increasing marginal disutility from working, (i.e., positive, but diminishing, marginal utility from leisure). Moreover, the instantaneous utility function $u$ is jointly strictly concave in $(c, l)$. In addition, the conditions stated in (2) ensure that consumption and leisure are normal goods.

In this simple framework we specify that individuals own the economy's physical capital, $k$, the services of which are rented to firms in a perfectly competitive capital market that yields a real return of $r$. In addition, the representative individual supplies $l$ units of labor services per unit of time and receives the real wage $w$, which is determined in a perfectly competitive labor market. Individuals can lend to and borrow from other individuals. Since physical capital and loans are assumed to be perfect substitutes as stores of value, they must pay the same real return of $r$. The flow budget constraint of the representative agent is then given by

$$
\dot{a}=r a+w l-c,
$$

where nonhuman wealth $a$ consists of physical capital $k$ and net loans $b$. We will assume that $k(0)=k_{0}>0$ and $b(0)=b_{0}=0$ so that $a(0)=k_{0}$, where $k_{0}$ is exogenously given

\footnotetext{
${ }^{2}$ See Frank (1985) for a discussion of positional goods.
} 
and positive. We further assume that the credit market imposes the following No-Ponzi Game (NPG) condition on the agent's borrowing:

$$
\lim _{t \rightarrow \infty}\left\{a(t) \exp \left[-\int_{0}^{t} r(v) d v\right]\right\} \geq 0 .
$$

Since agents in our model are identical in every respect, each holds zero net loans in any symmetric macroeconomic equilibrium, implying $a=k \geq 0$.

With respect to the production sector, we assume that there is a large number of perfectly competitive firms that rent the services of physical capital and labor to produce output. In addition, each firm has access to the same production possibilities. For convenience, we ignore in our subsequent analysis both depreciation of capital and technological progress. The production function is denoted by $y=F(k, l)$, where $y$ denotes output and is assumed to have the usual neoclassical properties of positive and diminishing marginal productivity of both factors, along with constant returns to scale. To consider whether the introduction of relative consumption or relative wealth into the instantaneous utility function leads to Pareto nonoptimality, we will compare the decentralized solutions with the solution from a hypothetical social planner's problem. We will begin with the social planner's problem that yields identical solutions for the two alternative specifications of status preferences.

\subsection{The Social Planner's Problem}

Suppose that there exists a benevolent social planner who dictates the choices of consumption and hours worked over time and who seeks to maximize the welfare of the representative individual. Since individuals are identical, we assume that the social planner assigns to each the same consumption level and the same level of work effort. Consequently, $c(t)=C(t), a(t)=A(t)$ and, thus, $z(t)=1$ hold for all $t$. Hence, the social planner's optimization problem can be written as follows: maximize intertemporal utility, equal to $\int_{0}^{\infty} e^{-\rho t} u(c, l, 1) d t$, by choosing the time paths of $c$ and $l$ subject to the economy's resource constraint

$$
\dot{k}=F(k, l)-c,
$$

and the initial condition $k(0)=k_{0}>0$, where $k_{0}$ is exogenously given. The current-value Hamiltonian for this problem is given by $H=u(c, l, 1)+\lambda[F(k, l)-c]$, where the costate variable $\lambda$ denotes the shadow price of capital. The necessary optimality conditions for an 
interior solution are given by $H_{c}=0, H_{l}=0$, and $\dot{\lambda}=\rho \lambda-H_{k}$ :

$$
\begin{gathered}
u_{c}(c, l, 1)-\lambda=0, \\
u_{l}(c, l, 1)+\lambda F_{l}(k, l)=0, \\
\dot{\lambda}=-\left[F_{k}(k, l)-\rho\right] \lambda .
\end{gathered}
$$

The transversality condition is given by

$$
\lim _{t \rightarrow \infty} e^{-\rho t} \lambda k=0
$$

The concavity assumptions made above ensure that if $(c, l, k)$ satisfies $(5)-(9)$ and the initial condition $k(0)=k_{0}$, then it is an optimal path. The properties of this solution with respect to its steady state and transitional dynamics will be studied in the next section.

\section{Decentralized Economy}

\subsection{General Solution}

If preferences are of the relative wealth type, then in the decentralized economy the representative individual chooses the time paths $c$ and $l$ to maximize $\int_{0}^{\infty} e^{-\rho t} u(c, l, a / A) d t$, subject to the flow budget constraint (3), the NPG-condition (4), and the initial condition $a(0)=k_{0}$. The representative agent takes not only the time paths of the rental rate of capital $r$ and the real wage rate $w$, but also the time path of average wealth $A$ as given. In order to obtain a well-behaved optimization problem in which the necessary conditions are also sufficient, we will assume that the function $U(c, l, a, A) \equiv u(c, l, a / A)$ is strictly concave in $(c, l, a)$. The current-value Hamiltonian of this optimization problem is $H=u(c, l, a / A)+\lambda(r a+w l-c)$. The necessary optimality conditions for an interior solution are given by $H_{c}=0, H_{l}=0$, and $\dot{\lambda}=\rho \lambda-H_{a}$ :

$$
\begin{gathered}
u_{c}(c, l, a / A)-\lambda=0, \\
u_{l}(c, l, a / A)+\lambda w=0, \\
\dot{\lambda}=-(r-\rho) \lambda-u_{z}(c, l, a / A) A^{-1}=-\left[r+\frac{u_{z}(c, l, a / A) A^{-1}}{u_{c}(c, l, a / A)}-\rho\right] \lambda .
\end{gathered}
$$

The transversality condition equals

$$
\lim _{t \rightarrow \infty} e^{-\rho t} \lambda a=0
$$


The concavity assumptions made above ensure that if $(c, l, a)$ satisfies (10)-(13), (3), and the initial condition $a(0)=k_{0}$, then it is an optimal path.

In (12), the expression $r+\left(u_{z} A^{-1}\right) / u_{c} \equiv r^{e}$ gives the effective return of wealth under relative wealth preferences. It is the sum of the market rate of return $r$ and the statusrelated component $\left(u_{z} A^{-1}\right) / u_{c}$, where the latter can be explained as follows. The fraction $u_{z} / u_{c}$ gives the marginal rate of substitution (MRS) of status, as measured by $z=a / A$, for consumption $c$. The expression $A^{-1}=z_{a}$ is the partial derivative of relative wealth with respect to own wealth. Hence, the status-related component $\left(u_{z} A^{-1}\right) / u_{c}$ is the MRS of own wealth $a$ for consumption $c$.

The next step in our analysis is to derive the symmetric macroeconomic equilibria for the decentralized economy. Since all individuals are identical, this means that: i) identical individuals make identical choices so that $a=A$ and $z \equiv a / A=1$ for all $t$; ii) each individual holds zero net loans so that net wealth is simply equal to the capital stock, i.e., $a=k$; iii) the real rental rate and the real wage are determined by the profit-maximizing conditions $r=F_{k}(k, l)$ and $w=F_{l}(k, l)$; and iv) the constant returns to scale assumption implies that $F(k, l)=r k+w l$. In a symmetric macroeconomic equilibrium the dynamic evolution of $(c, l, k, \lambda)$ is determined by the conditions for the optimal choice of $c$ and $l$, equations (6) and (7), the economy's flow resource constraint (5), the differential equation

$$
\dot{\lambda}=-\left[F_{k}(k, l)+\frac{u_{z}(c, l, 1) k^{-1}}{u_{c}(c, l, 1)}-\rho\right] \lambda
$$

that governs the dynamic evolution of the shadow price $\lambda$, the transversality condition (9) and the initial condition $k(0)=k$. Note that these equations and conditions are identical with those that determine the socially optimal solution, with the single exception that (8), $\dot{\lambda}=-\left[F_{k}(k, l)-\rho\right] \lambda$, is replaced by (14). This means that in the relative wealth context the equilibrium effective rate of return, $F_{k}+\left(u_{z} / u_{c}\right) k^{-1}$, exceeds the market rate of return, $F_{k}$, which, in turn, implies that the decentralized solution is inefficient.

As we discussed in the introduction, one of our major goals in this paper is to contrast the implications of the relative wealth preferences with those of relative consumption. Since the relative consumption approach has been dealt with in detail by authors such as Fisher and Hof (2000b) and Liu and Turnovsky (2005), we will employ their main results without reproducing their analysis. In the socially planned economy the marginal rate of substitution of consumption for leisure as perceived by the social planner, who takes into account the externalities, is given by $(M R S)^{p}(c, l)=u_{c}(c, l, 1) /\left[-u_{l}(c, l, 1)\right]$, 
where the superscript $p$ stands for "(social) planner". Under relative wealth preferences, in a symmetric equilibrium the MRS of consumption for leisure as perceived by the representative consumer who takes the time path of $A$ as given equals $(M R S)^{d, r w}(c, l)=$ $u_{c}(c, l, 1) /\left[-u_{l}(c, l, 1)\right]$, where the superscripts $d$ and $r w$ stand, respectively, for "decentralized (economy)" and "relative wealth." From $(M R S)^{p}(c, l)=(M R S)^{d, r w}(c, l)$ it is clear that wealth externalities do not (directly) distort the choice of consumption and work effort. Instead, as is evident from the costate equation (14), they distort the dynamic evolution of the economy by raising the decentralized rate of return of wealth, $F_{k}+\left(u_{z} / u_{c}\right) k^{-1}$, above its socially optimal counterpart, $F_{k}$.

If preferences are of the relative consumption type, then the differential equation governing the dynamic evolution of the shadow value of wealth is the same as in the socially planned economy, $\dot{\lambda}=-\left[F_{k}(k, l)-\rho\right] \lambda$. In other words, the decentralized rate of return is not distorted in a direct way if status depends on relative consumption. Instead, consumption externalities raise the willingness to substitute consumption for leisure above its socially optimal level. More specifically, in a symmetric equilibrium in which $c=C$, the decentralized MRS as perceived by the representative consumer corresponds to

$$
(M R S)^{d, r c}(c, l)=\frac{u_{c}(c, l, 1)+c^{-1} u_{z}(c, l, 1)}{-u_{l}(c, l, 1)}>(M R S)^{p}(c, l),
$$

where the superscript $r c$ stands for "relative consumption". Observe that the expression $u_{c}+c^{-1} u_{z}>0$ measures the total marginal utility of own consumption. Because agents take the time path of $C$ as given, they believe that an increase in $c$ would also result in a rise in relative consumption (and status). The term $c^{-1} u_{z}$ measures the perceived additional gain in utility. Of course, because all agents act in this way, no one agent succeeds in raising relative consumption. In Fisher and Hof (2000b) it is shown that the distortions arising from consumption externalities can be eliminated by imposing an appropriate consumption $\operatorname{tax}$.

Finally, under relative consumption preferences some properties of the steady state are easily obtained by using the fact that the differential equations $\dot{\lambda}=-\left[F_{k}(k, l)-\rho\right] \lambda$ and $\dot{k}=F(k, l)-c$ are common to both the decentralized and the socially planned economies. Since, in addition, the production function exhibits constant returns to scale, it is clear that the decentralized steady-state value of the capital-labor ratio, $k / l$, equals its socially optimal counterpart, determined by $F_{k}(k / l, 1)=\rho$. Nevertheless, while the decentralized 
ratios $k / l$ and $c / l$ are optimal in the long run, the decentralized levels of $c, l$ and $k$ are not. Under plausible assumptions with respect to preferences, it is straightforward to show [see, for example, Fisher and Hof (2000b) and Liu and Turnovsky (2005)] that in longrun equilibrium agents work and consume too much, and excessively accumulate physical capital.

Having reviewed some of the basic properties of the relative consumption model, we continue with our analysis of the relative wealth specification. First, we will show that the characteristics of the steady state differ significantly from those resulting from the relative consumption specification. To do so, it is convenient to rewrite the production function in the intensive form $F(k, l)=l f(\kappa)$, where $\kappa \equiv k / l$ denotes the capital-labor ratio and $f(\kappa) \equiv F(\kappa, 1)$. This notation implies that $F_{k}(k, l)=f^{\prime}(\kappa)$ and $F_{l}(k, l)=f(\kappa)-\kappa f^{\prime}(\kappa)$. In the decentralized economy the steady-state values of consumption, work effort, and the capital-labor ratio, denoted by $\tilde{c}^{d}, \tilde{l}^{d}$, and $\tilde{\kappa}^{d}$, are determined by

$$
\begin{gathered}
c=l f(\kappa), \\
\frac{-u_{l}(c, l, 1)}{u_{c}(c, l, 1)}=f(\kappa)-\kappa f^{\prime}(\kappa), \\
0=f^{\prime}(\kappa)+\frac{u_{z}(c, l, 1)}{u_{c}(c, l, 1)} \frac{1}{\kappa l}-\rho .
\end{gathered}
$$

In the socially planned economy the corresponding steady-state values, $\tilde{c}^{p}, \tilde{l}^{p}$, and $\tilde{\kappa}^{p}$, are determined by (16), (17), while (18) is replaced by

$$
f^{\prime}(\kappa)=\rho .
$$

To derive relationships that enable us to compare the decentralized to the socially optimal steady state, we first we solve equations (16) and (17) implicitly for $c$ and $l$ in terms of $\kappa$ :

$$
\begin{array}{ll}
c=h^{c}(\kappa), & h_{\kappa}^{c}=\frac{\left(u_{c} u_{l l}-u_{c l} u_{l}\right) l f^{\prime}+f u_{c}^{2} \kappa f^{\prime \prime}}{\left(u_{c} u_{l l}-u_{c l} u_{l}\right)+\left(u_{l c} u_{c}-u_{c c} u_{l}\right) f}, \\
l=h^{l}(\kappa), & h_{\kappa}^{l}=\frac{u_{c}^{2} \kappa f^{\prime \prime}-\left(u_{l c} u_{c}-u_{c c} u_{l}\right) l f^{\prime}}{\left(u_{c} u_{l l}-u_{c l} u_{l}\right)+\left(u_{l c} u_{c}-u_{c c} u_{l}\right) f} .
\end{array}
$$

Using, in turn, equation (21) and the relationship $k=\kappa l$, we obtain the following solution for the capital stock $k$ :

$$
k=h^{k}(\kappa), \quad h_{\kappa}^{k}=\frac{\left(u_{c} u_{l l}-u_{c l} u_{l}\right) l+\left(u_{l c} u_{c}-u_{c c} u_{l}\right) l\left(f-\kappa f^{\prime}\right)+u_{c}^{2} \kappa^{2} f^{\prime \prime}}{\left(u_{c} u_{l l}-u_{c l} u_{l}\right)+\left(u_{l c} u_{c}-u_{c c} u_{l}\right) f} .
$$


In (20)-(22) $l=h^{l}(\kappa)$ and the partial derivatives of $u$ are evaluated at $\left(h^{c}(\kappa), h^{l}(\kappa), 1\right)$. Taking into account that $u_{c} u_{l l}-u_{c l} u_{l}<0$ and $u_{l c} u_{c}-u_{c c} u_{l}<0$ hold due to the normality conditions given in (2) and that $f^{\prime}>0, f^{\prime \prime}<0$, and $f-\kappa f^{\prime}>0$, it is obvious that $h_{\kappa}^{c}>0$ and $h_{\kappa}^{k}>0$, while, in contrast, the sign of $h_{\kappa}^{l}$ cannot be determined unambiguously. Since (16) and (17) and thus (20)-(22) hold both in the socially planned economy and the decentralized economy, we obtain

$$
\begin{aligned}
\tilde{c}^{d}=h^{c}\left(\tilde{\kappa}^{d}\right), & \tilde{l}^{d}=h^{l}\left(\tilde{\kappa}^{d}\right), & \tilde{k}^{d}=h^{k}\left(\tilde{\kappa}^{d}\right), \\
\tilde{c}^{p}=h^{c}\left(\tilde{\kappa}^{p}\right), & \tilde{l}^{p}=h^{l}\left(\tilde{\kappa}^{p}\right), & \tilde{k}^{p}=h^{k}\left(\tilde{\kappa}^{p}\right) .
\end{aligned}
$$

While the functions $h^{c}, h^{l}$, and $h^{k}$ are identical in both economies, the corresponding steady-state values are not. From (18) and (19) it follows that

$$
f^{\prime}\left(\tilde{\kappa}^{d}\right)=f^{\prime}\left(\tilde{\kappa}^{p}\right)-\frac{u_{z}\left(\tilde{c}^{d}, \tilde{l}^{d}, 1\right)}{u_{c}\left(\tilde{c}^{d}, \tilde{l}^{d}, 1\right)} \frac{1}{\tilde{\kappa}^{d} \tilde{l}^{d}}<f^{\prime}\left(\tilde{\kappa}^{p}\right) .
$$

From $f^{\prime}\left(\tilde{\kappa}^{d}\right)<f^{\prime}\left(\tilde{\kappa}^{p}\right)$ and $f^{\prime \prime}<0$, it is clear that any steady-state value $\tilde{\kappa}^{d}$ satisfies the condition $\tilde{\kappa}^{d}>\tilde{\kappa}^{p}$, implying that the long-run capital-labor ratio is higher in the decentralized equilibrium. Observe, however, that uniqueness of the steady state is not a general property of the relative wealth framework [see e.g. Corneo and Jeanne (2001a), Hof and Wirl (2003)]. From (16) and $f^{\prime}>0$, it then follows that $\tilde{c}^{d} / \tilde{l}^{d}>\tilde{c}^{p} / \tilde{l}^{p}$. Moreover, taking into account that $h_{\kappa}^{c}>0$ and $h_{\kappa}^{k}>0$, we obtain $\tilde{c}^{d}>\tilde{c}^{p}$ and $\tilde{k}^{d}>\tilde{k}^{p}$, i.e., individuals consume too much and accumulate an excessive stock of capital in steady-state equilibrium. On the other hand, since the sign of $h_{\kappa}^{l}$ cannot be determined unambiguously, we cannot infer from this analysis whether $\tilde{l}^{d}>\tilde{l}^{p}$ or $\tilde{l}^{d} \leq \tilde{l}^{p}$ holds. Below we will show by means of an illustration that unlike the relative consumption approach, it is indeed possible that the steady-state level of work effort is less than or equal to its socially optimal counterpart and that this steady state is sensible, since it has the desirable saddlepoint property.

In order to study the stability properties of the steady states in both the decentralized economy and the socially planned one, we could apply the standard procedures described in detail in Turnovsky (1995) to our general model. While the analysis of the socially planned economy yields the result that the corresponding unique steady state exhibits the saddlepoint property, a similar result cannot be obtained in the decentralized economy without imposing many additional assumptions with respect to the partial derivatives $u_{z}$, $u_{z l}$, and $u_{z c}$. For this reason, we will subsequently restrict our attention to a simple illustration. On the one hand, this particular specification yields a unique, saddlepoint-stable 
steady state. On the other hand, it is sufficiently general to allow for both a wide variety of transitional dynamics and the steady-state properties of work effort. For example, whether agents work "too little" or "too much" in the long run, depends on the value of the intertemporal elasticity of substitution for consumption.

\subsection{Illustration}

We assume that the instantaneous utility function takes the form

$$
u(c, l, z)=(1-\theta)^{-1}\left[\left(c z^{\beta}\right)^{1-\theta}-1\right]-\mu l^{1+\sigma},
$$

where $\beta \geq 0, \sigma>0, \theta>0, \mu>0$, and $\beta(\theta-1)+\theta>0$. These assumptions ensure that the function $U(c, l, a, A) \equiv u(c, l, a / A)$ is jointly concave in $(c, l, a)$. Moreover, we assume that the production function takes the standard Cobb-Douglas specification

$$
F(k, l)=B k^{\alpha} l^{1-a}, \quad B>0, \quad 0<\alpha<1 .
$$

Our specifications imply that the optimization problems of both the representative agent in the decentralized economy and the social planner are well-behaved, in the sense that i) we obtain interior solutions, and ii) if the transversality condition holds, then the necessary optimality conditions are also sufficient. The dynamic evolution of $(c, l, k, \lambda)$ in the decentralized economy is governed by

$$
\begin{gathered}
\dot{k}=B k^{\alpha} l^{1-\alpha}-c, \\
c^{-\theta}-\lambda=0, \\
-\mu(1+\sigma) l^{\sigma}+\lambda(1-\alpha) B(k / l)^{\alpha}=0, \\
\dot{\lambda}=-\left[\alpha B(k / l)^{-(1-\alpha)}+\beta(c / k)-\rho\right] \lambda,
\end{gathered}
$$

the initial condition $k(0)=k_{0}$, and the transversality condition $\lim _{t \rightarrow \infty} e^{-\rho t} \lambda k=0 .{ }^{3}$ The corresponding system for the socially planned economy is obtained by replacing (30) with

$$
\dot{\lambda}=-\left[\alpha B(k / l)^{-(1-\alpha)}-\rho\right] \lambda .
$$

\footnotetext{
${ }^{3}$ Under (25) the MRS of status $z$ for consumption $c, u_{z} / u_{c}$, equals $\beta(c / z)$. In a symmetric equilibrium this expression simplifies to $\beta c$ and, moreover, the partial derivative of relative wealth with respect to own wealth is given by $z_{a}=k^{-1}$. Hence, in a symmetric equilibrium the MRS of own wealth $a$ for consumption $c$ is given by $\beta(c / k)$.
} 
Observe that under the preference specification (25), the system of equations for the socially planned economy does not depend on the status parameter $\beta$. Both the system that governs the dynamic evolution of the socially planned economy and its solutions are simply obtained by setting $\beta=0$ in the corresponding equations of the decentralized economy. ${ }^{4}$ Consequently, the steady-state values $\tilde{\kappa}, \tilde{l}, \tilde{c}$, and $\tilde{k}$ that are given in appendix 5.1 must be interpreted as follows: $\tilde{x}^{p}=\left.\tilde{x}\right|_{\beta=0}$ and $\tilde{x}^{d}=\left.\tilde{x}\right|_{\beta>0}$. Using these solutions, the signs of the long-run multipliers with respect to the status parameter $\beta$ are determined as follows: $\partial \tilde{\kappa} / \partial \beta>0, \partial \tilde{c} / \partial \beta>0, \partial \tilde{k} / \partial \beta>0, \operatorname{sgn}(\partial \tilde{l} / \partial \beta)=\operatorname{sgn}(1-\theta)$. From these results it is obvious that the steady-state values of the capital-labor ratio, consumption, and physical capital depend positively on the status parameter $\beta$. Hence, compared to the socially planned economy, not only is the capital-labor ratio "too high", $\tilde{\kappa}^{d}>\tilde{\kappa}^{p}$, but agents also consume "too much", $\tilde{c}^{d}>\tilde{c}^{p}$, and accumulate an "excessive" stock of capital in the decentralized economy, $\tilde{k}^{d}>\tilde{k}^{p}$. Moreover, the marginal product of labor and, hence, the real wage exceed their socially optimal counterparts, $\tilde{F}_{l}^{d}>\tilde{F}_{l}^{p}$, while the opposite result holds for the rental rate of capital, $\tilde{F}_{k}^{d}<\tilde{F}_{k}^{p}=\rho$. Note, however, that $\partial \tilde{l} / \partial \beta$ may be of either sign. If $\theta<1$, then agents work too much in the long run, $\tilde{l}^{d}>\tilde{l}^{p}$. On the other hand, if $\theta>1$, then the steady-state value of work effort is less than its socially optimal counterpart, $\tilde{l}^{d}<\tilde{l}^{p}$. The question arises why agents are worse off in the decentralized economy when, in addition to $\tilde{c}^{d}>\tilde{c}^{p}$, it is the case that $\tilde{l}^{d}<\tilde{l}^{p}$ if $\theta>1$. In other words, are agents, in fact, worse off compared to the social optimum if they consume more and enjoy more leisure in the long run? The answer is that intertemporal utility does not only depend on the long-run values of consumption and work effort, but is also a function of the transitional dynamics of these variables. The excessive capital accumulation requires that there is a time interval with excessive saving. Under the specific preferences given by (25), excessive saving, in turn, is achieved by both under-consumption and excessive work effort.

Next, we will study the stability properties of the steady state and the transitional

\footnotetext{
${ }^{4}$ This property does not hold in general. For instance, if the utility function is of the of Harbaugh (1996) type,

$$
u=(1-\theta)^{-1}\left[\left(c^{1-\beta} z^{\beta}\right)^{1-\theta}-1\right]-\mu l^{1+\sigma}, \quad \theta>0, \mu>0, \sigma>0,0<\beta<1,
$$

then both the decentralized and the socially optimal solution depend on $\beta$. It is not possible to obtain the socially optimal solution simply by setting $\beta=0$ [for details see Hof (2004)].
} 
dynamics. Note the following analysis applies to both the decentralized economy and the socially planned one. From (27)-(30) three alternative representations of the nonlinear differential equation system can be derived: $\dot{k}=\dot{k}(k, x)$ and $\dot{x}=\dot{x}(k, x)$, where $x=c$, $l, \lambda$. In order to obtain the system of differential equations $\dot{k}=\dot{k}(k, c)$ and $\dot{c}=\dot{c}(k, c)$ [resp. $\dot{k}=\dot{k}(k, l)$ and $\dot{l}=\dot{i}(k, l)]$, on which the control-state-space analysis is based, equations (28) and (29) are first solved for $l$ and $\lambda$ as functions of $k$ and $c$ [resp. for $c$ and $\lambda$ as functions of $k$ and $l]$ and then the resulting expressions are substituted into the differential equations (27) and (30). Alternatively, if the analysis is carried out in the state-costate space, two differential equations of the form $\dot{k}=\dot{k}(k, \lambda)$ and $\dot{\lambda}=\dot{\lambda}(k, \lambda)$ are obtained by first solving the necessary optimality conditions (28) and (29) for $c$ and $l$ as functions of $k$ and $\lambda$. In this paper will focus our attention on the control-state-space analysis, since we can directly see the time paths of consumption and employment in the corresponding phase diagrams. Linearization around the steady states $(\tilde{k}, \tilde{x}), x=\lambda, c, l$, yields

$$
\left(\begin{array}{c}
\dot{k} \\
\dot{x}
\end{array}\right)=\mathbf{M}^{x}\left(\begin{array}{c}
k-\tilde{k} \\
x-\tilde{x}
\end{array}\right), \quad \mathbf{M}^{x} \equiv\left(\begin{array}{cc}
\frac{\partial \dot{k}}{\partial k}(\tilde{k}, \tilde{x}) & \frac{\partial \dot{k}}{\partial x}(\tilde{k}, \tilde{x}) \\
\frac{\partial \dot{x}}{\partial k}(\tilde{k}, \tilde{x}) & \frac{\partial \dot{x}}{\partial x}(\tilde{k}, \tilde{x})
\end{array}\right),
$$

where $\mathbf{M}^{x}, x=\lambda, c, l$, is the corresponding Jacobian. Its elements are denoted by $m_{i j}^{x}$, $i, j=1,2 ; x=\lambda, c, l$. While the $m_{i j}^{x}$ depend on whether $x$ represents $\lambda, c$, or $l$, the traces and the determinants of $\mathbf{M}^{\lambda}, \mathbf{M}^{c}$ and $\mathbf{M}^{l}$ are identical $\left(\mathbf{M}^{c}\right.$ and $\mathbf{M}^{l}$ are given in appendix 5.2.1 and 5.3.3). The common characteristic equation is given by

$$
0=P(\xi) \equiv \xi^{2}-\frac{(\alpha \theta+\beta) \rho}{(\alpha+\beta) \theta} \xi-\frac{(1-\alpha)(\sigma+\theta) \rho^{2}}{\theta(\alpha+\beta)(\alpha+\sigma)} .
$$

Observe that the common determinant of the Jacobians, given by last term in (32), is negative, so that the roots of the characteristic equation are of opposite sign, i.e., $\xi_{1}<0$ and $\xi_{2}>0$. Since this holds for $\beta \geq 0$, in both the decentralized and the socially planned economies, the steady states exhibit saddlepoint stability. Before turning to a detailed analysis of the economy's transitional dynamics, we summarize our results thus far.

Proposition 1 If status is determined by relative wealth, then

A) contrary to the relative consumption approach, any steady-state value of the capitallabor ratio and the consumption-labor ratio in the decentralized economy exceeds its socially optimal counterpart, i.e., $\tilde{k}^{d} / \tilde{l}^{d}>\tilde{k}^{p} / \tilde{l}^{p}$ and $\tilde{c}^{d} / \tilde{l}^{d}>\tilde{c}^{p} / \tilde{l}^{p}$, 
B) as in the relative consumption framework, individuals in the long run consume too much and accumulate an excessive stock of capital, i.e., $\tilde{c}^{d}>\tilde{c}^{p}$ and $\tilde{k}^{d}>\tilde{k}^{p}$,

C) contrary to the relative consumption approach in which individuals always work too much in the long run, the steady-state level of work effort can be less than or equal to its socially optimal counterpart,

D) there are specifications of preferences and technology that result in unique steady states in both the decentralized economy and the socially planned one that have the desirable saddlepoint property.

The proof of this proposition follows from the analysis given above.

\subsubsection{Consumption Dynamics}

We now turn to the analysis of the transitional dynamics. First, we investigate the behavior of consumption, using the phase diagram in the $(k, c)$ plane depicted in Figure 1. Solving the necessary optimality conditions (28) and (29) for $l$ and $\lambda$ we obtain:

$$
l=\left(\frac{(1-\alpha) B}{\mu(1+\sigma)}\right)^{\frac{1}{\alpha+\sigma}} k^{\frac{\alpha}{\alpha+\sigma}} c^{-\frac{\theta}{\alpha+\sigma}}, \quad \lambda=c^{-\theta} .
$$

Note that $l$ depends positively on capital $k$ and negatively on consumption $c$. The latter effect means that for a given level of $k$, leisure and consumption always move in the same direction as $c$ changes. Substitution of (33) into (27) and (30) then yields the following system:

$$
\begin{gathered}
\dot{k}=\left(\frac{1-\alpha}{\mu(1+\sigma)}\right)^{\frac{1-\alpha}{\alpha+\sigma}} B^{\frac{1+\sigma}{\alpha+\sigma}} k^{\frac{\alpha(1+\sigma)}{\alpha+\sigma}} c^{-\frac{\theta(1-\alpha)}{\alpha+\sigma}}-c \\
\dot{c}=\frac{c}{\theta}\left[\alpha\left(\frac{1-\alpha}{\mu(1+\sigma)}\right)^{\frac{1-\alpha}{\alpha+\sigma}} B^{\frac{1+\sigma}{\alpha+\sigma}} k^{-\frac{(1-\alpha) \sigma}{\alpha+\sigma}} c^{-\frac{(1-\alpha) \theta}{\alpha+\sigma}}+\beta(c / k)-\rho\right] .
\end{gathered}
$$

The resulting $\dot{k}=0$ isocline, depicted in Figure 1,

$$
\left.c\right|_{\dot{k}=0}=\left(\left(\frac{1-\alpha}{\mu(1+\sigma)}\right)^{1-\alpha} B^{1+\sigma} k^{\alpha(1+\sigma)}\right)^{\frac{1}{\alpha+\sigma+\theta(1-\alpha)}},
$$

is positively sloped, strictly concave, and independent of the status parameter $\beta$. To the right of $\dot{k}=0$, production exceeds consumption so that $\dot{k}>0$ holds. Note that this rise in production is due not only to the rise in capital $k$, but also to the implied increase in 
employment $l$ [see (33)]. Similarly, to the left of $\dot{k}=0$ production falls short of consumption so that $\dot{k}<0$.

As an aid to intuition, it is convenient to write the Euler equation for consumption $(35)$ as

$$
\dot{c}=\theta^{-1} c\left[r^{e}(k, c, \beta)-\rho\right], \quad \text { where } \quad r^{e}(k, c, \beta) \equiv F_{k}(\kappa(k, c), 1)+\beta(c / k)
$$

denotes the effective rate of return, which consists of the market rate of return $F_{k}$ and the rate of return due to relative wealth preferences $\beta(c / k)$, such that the capital-labor ratio $\kappa$, using (33), is expressed as a function of $k$ and $c$ :

$$
\kappa(k, c) \equiv \frac{k}{l(k, c)}=\left(\frac{(1-\alpha) B}{\mu(1+\sigma)}\right)^{-\frac{1}{\alpha+\sigma}} k^{\frac{\sigma}{\alpha+\sigma}} c^{\frac{\theta}{\alpha+\sigma}} .
$$

It is clear that $\kappa_{k}>0$ and $\kappa_{c}>0$ : if $k$ rises by 1 percent, then $l(k, c)$ rises by less than 1 percent, which, in turn, implies that the capital-labor ratio depends positively on $k$. A rise in optimal consumption is accompanied by a rise in leisure, i.e., a decrease in work effort. Therefore, the capital-labor ratio depends positively on $c$.

For $c>0$, the $\dot{c}=0$ isocline is implicitly determined by the equality of the effective rate of return and the subjective discount rate, $r^{e}(k, c, \beta)=\rho$. In the case $\beta=0$, which also corresponds to the socially planned economy, this condition simplifies to $F_{k}(\kappa(k, c), 1)=$ $\rho$, implying that the capital-labor ratio $\kappa(k, c)$ is constant along $\dot{c}=0$. From $\kappa_{k}>0$ and $\kappa_{c}>0$, it is clear that any rise in $c$ must be offset by a decrease in $k$, i.e., the $\dot{c}=0$ isocline is negatively sloped and strictly convex, as illustrated in Figure 1. These properties, due solely to the fact that work effort is endogenous, are in contrast to those of the textbook Ramsey model with exogenous labor supply in which $\dot{c}=0$ is a vertical line in the $(k, c)$ plane.

In the decentralized economy in which $\beta>0$, the properties of the $\dot{c}=0$ isocline are more complex. Note first that the partial derivative of the effective rate of return with respect to capital is negative, $r_{k}^{e}<0$, The reason is that a rise in $k$ : i) causes the market rate of return $F_{k}$ to decrease due to the implied rise in the capital-labor ratio $\kappa(k, c)$, and ii) leads to a fall in the status-dependent component of $r^{e}$ given by $\beta(c / k)$. In contrast, the partial derivative with respect to consumption, $r_{c}^{e}$, may be of either sign. On the one hand, a rise in $c$ decreases $F_{k}$ by raising the capital-labor ratio $\kappa(k, c)$, while, on the other hand, it leads to an increase in $\beta(c / k)$. These properties of $r_{k}^{e}$ and $r_{c}^{e}$ mean that the condition 
$\dot{c}=0$ determines implicitly $k$ as a function of $c$ and $\beta$. From $r_{k}^{e}<0$ and $r_{\beta}^{e}>0$, it is also evident that a rise in the status parameter $\beta$ causes the $\dot{c}=0$ isocline, as shown in Figure 1, to shift to the right. Regardless of the value of $\beta$, it follows from $r_{k}^{e}<0$ that to the right of the $\dot{c}=0$ isocline the effective rate of return is less than the subjective discount rate, $r^{e}<\rho$. Therefore, according to the Euler relationship, agents then choose a declining path of consumption, $\dot{c}<0$. Similarly, to the left of the $\dot{c}=0$ isocline $r^{e}>\rho$, so that $\dot{c}>0$. Comparing the steady states at points $P$ and $D$ in Figure 1 , which correspond, respectively, to the socially planned, $\beta=0$, and decentralized economies, $\beta>0$, it is clear that agents in the decentralized equilibrium consume too much in the long run and accumulate too much capital. ${ }^{5}$ This confirms result B) of proposition 1.

Now let us turn to a detailed analysis of the economy's transitional dynamics. Employing both the initial condition $k(0)=k_{0}$ and the transversality condition, one can obtain the following general representation of the stable saddle paths in the $(k, x)$ plane, where $x=c, l$ :

$$
x(t)-\tilde{x}=\frac{m_{11}^{x}-\xi_{1}}{-m_{12}^{x}}(k(t)-\tilde{k})=\frac{-m_{21}^{x}}{m_{22}^{x}-\xi_{1}}(k(t)-\tilde{k}),
$$

where the dynamic evolution of $k$ is governed by

$$
k(t)=\tilde{k}+\left(k_{0}-\tilde{k}\right) e^{\xi_{1} t} .
$$

The last equality in (36) makes use of the fact that the characteristic equation $0=P(\xi)$ can be written as $0=\left(m_{11}^{x}-\xi\right)\left(m_{22}^{x}-\xi\right)-m_{12}^{x} m_{21}^{x}$. Since $m_{11}^{c}>0, m_{12}^{c}<0$, and $\xi_{1}<0$, where the expressions for $m_{11}^{c}$ and $m_{12}^{c}$ are given in appendix 5.2.1, it follows from (36) that the stable arm in the $(k, c)$ plane is positively sloped, as depicted in Figure 1. In other words, capital and consumption always move in the same direction. Observe that this qualitative result holds for both the decentralized economy in which preferences are of the relative wealth type for $\beta>0$ and for the socially planned economy, the solutions of which are obtained by setting $\beta=0$. While $\beta$ does not affect the sign of the slope of a stable saddle path, variations in the value of $\beta$ lead to shifts of the stable arm and can also cause the magnitude of its slope to change. A special focus of our analysis is how the initial values of consumption $c(0)$ and work effort $l(0)$ depend on $\beta$. Using the first

\footnotetext{
${ }^{5}$ Using the results for $m_{21}^{c}$ and $m_{22}^{c}$ given in appendix 5.2.1, it can be shown that the $\dot{c}=0$ locus for $\beta>0$ is negatively sloped at the point of intersection $D$ with the $\dot{k}=0$ locus if and only if $\beta<$ $\alpha(1-\alpha) \theta(\alpha+\sigma)^{-1}$.
} 
equality in (36), setting $t=0$ and differentiating with respect to $\beta$ we obtain:

$$
\frac{\partial x(0)}{\partial \beta}=\left[\frac{\partial \tilde{x}}{\partial \beta}-\frac{m_{11}^{x}-\xi_{1}}{-m_{12}^{x}} \frac{\partial \tilde{k}}{\partial \beta}\right]+\left(k_{0}-\tilde{k}\right) \frac{\partial\left[\left(m_{11}^{x}-\xi_{1}\right) /\left(-m_{12}^{x}\right)\right]}{\partial \beta} .
$$

An analogous result is obtained by employing the second equality in (36). The term in square brackets, the shift effect, describes the reaction of $x(0)$ resulting from a parallel shift of the stable arm, which is due to the changes in the steady-state values $\tilde{x}$ and $\tilde{k}$. The other term, slope effect, captures the reaction of $x(0)$ that is due to the change in the magnitude of the stable arm's slope. Note that the slope depends also on the negative root $\xi_{1}$, which determines the speed of convergence to the long-run equilibrium. In appendix 5.2.2 it is shown that

$$
\partial\left|\xi_{1}\right| / \partial \beta<0
$$

for all admissible parameter values. In other words, an increase in the status parameter $\beta$ "slows down" the speed of convergence along stable saddle path. There, it is also shown analytically by evaluating (38) for $x=c$ that a rise in $\beta$ causes the stable arm in the $(k, c)$ plane to i) shift downwards, and ii) to become flatter. ${ }^{6}$ The shift effect leads to a decline in initial consumption $c(0)$. If $k_{0}<\tilde{k}$ obtains, an assumption that we maintain for the rest of the paper, then the slope effect causes $c(0)$ to increase. Nevertheless, if $k_{0}$ is sufficiently close to $\tilde{k}$, the negative shift effect will dominate the positive slope effect so that $c(0)$ falls. In other words, initial consumption in the decentralized economy with status preferences is "too small" compared to its socially optimal value, i.e., $\left.c(0)\right|_{\beta>0}<\left.c(0)\right|_{\beta=0}$.

\subsubsection{Employment Dynamics}

We now consider the behavior of work effort, using the phase diagram analysis in the $(k, l)$ plane. Solving the necessary optimality conditions (28) and (29) for $c$ and $\lambda$ we obtain:

$$
c=\left(\frac{(1-\alpha) B}{\mu(1+\sigma)}\right)^{\frac{1}{\theta}} k^{\frac{\alpha}{\theta}} l^{-\frac{\alpha+\sigma}{\theta}}, \quad \lambda=\frac{\mu(1+\sigma)}{(1-\alpha) B} k^{-\alpha} l^{\alpha+\sigma} .
$$

Substituting the expression for $c$ into (27) yields

$$
\dot{k}=B k^{\alpha} l^{1-\alpha}-\left(\frac{(1-\alpha) B}{\mu(1+\sigma)}\right)^{\frac{1}{\theta}} k^{\frac{\alpha}{\theta}} l^{-\frac{\alpha+\sigma}{\theta}} .
$$

\footnotetext{
${ }^{6}$ Observe that while the phase diagram depicted in Figure 1 offers a graphical proof for this downward shift of the stable arm, the change in its slope can only be determined analytically.
} 
With respect to the $\dot{k}=0$ isocline, which is given by

$$
l_{\dot{k}=0}=\left(\frac{(1-\alpha) B^{1-\theta}}{\mu(1+\sigma)}\right)^{\frac{1}{\alpha+\sigma+(1-\alpha) \theta}} k^{\frac{\alpha(1-\theta)}{\alpha+\sigma+(1-\alpha) \theta}}
$$

we distinguish between the following three cases: i) if $\theta=1$, then it is a horizontal line in the $(k, l)$ plane; ii) if $\theta<1$, then it is positively sloped and strictly concave; and iii) if $\theta>1$, then it is negatively sloped and strictly convex.

From (41) it is clear that $\partial \dot{k} / \partial l>0$, which, in turn, implies that $\dot{k}>0$ above the $\dot{k}=0$ isocline. The economic interpretation of $\partial \dot{k} / \partial l>0$ is straightforward. An increase in $l$ causes production to rise and is accompanied by a decrease in optimal consumption [see (40)]. Similarly, below the $\dot{k}=0$ isocline $\dot{k}<0$ holds.

In order to obtain the differential equation for work effort, we first differentiate the solution for $\lambda$ given in (40) with respect to time $t$, and then substitute the resulting expression for $\dot{\lambda}$ as well as the solutions for $c$ and $\lambda$ into (30):

$$
i / l=\frac{1}{\alpha+\sigma}\left\{\alpha\left(\frac{\dot{k}}{k}\right)-\left[\alpha B\left(\frac{k}{l}\right)^{-(1-\alpha)}+\beta\left(\frac{(1-\alpha) B}{\mu(1+\sigma)}\right)^{\frac{1}{\theta}} k^{\frac{\alpha-\theta}{\theta}} l^{-\frac{\alpha+\sigma}{\theta}}\right]+\rho\right\} .
$$

This differential equation can interpreted as follows: in appendix 5.3.1 we show that if the utility function $u(c, l, z)$ takes the general additively separable form $U(c, z)+V(l)$, then the necessary optimality conditions (10) and (11) can be solved for $c$ and $\lambda$ in the form $c=\hat{c}(w, l, a / A)$ and $\lambda=\hat{\lambda}(w, l)$. Moreover, the corresponding Euler equation for the agent's labor supply becomes

$$
\dot{l} / l=\frac{V^{\prime}}{l V^{\prime \prime}}\left[\frac{\dot{w}}{w}-\left(r+\frac{U_{z} A^{-1}}{U_{c}}\right)+\rho\right],
$$

where the partial derivatives of $U$ are evaluated at $(c, z)=(\hat{c}(w, l, a / A), a / A)$. It is clear from the Euler equation that agents choose a rising path of work effort when the sum of the growth rate of wages and the subjective discount rate exceeds the effective rate of return. This representation corresponds to the idea of intertemporal substitution of leisure, where, in contrast to the standard model, the market rate of return $r$ is replaced by the effective rate of return $r^{e}$. Under our specific parameterization (25), the expression $V^{\prime} /\left(l V^{\prime \prime}\right)$, which gives the intertemporal elasticity of the agent's labor supply, simplifies to $\sigma^{-1}$.

In a symmetric macroeconomic equilibrium $a=A=K=k, r=F_{k}(k, l)$ and $w=F_{l}(k, l)$ hold. Using these relationships, the previous expression for the Euler equa- 
tion for individual labor supply yields the following differential equation for equilibrium employment (details are found in appendix 5.3.2):

$$
i / l=\left(\frac{l V^{\prime \prime}}{V^{\prime}}-\frac{l F_{l l}}{F_{l}}\right)^{-1}\left[\frac{k F_{l k}}{F_{l}}\left(\frac{\dot{k}}{k}\right)-\left(F_{k}+\frac{U_{z} k^{-1}}{U_{c}}\right)+\rho\right],
$$

where the partial derivatives of $U$ are evaluated at $(c, z)=\left(\hat{c}\left(F_{l}(k, l), l, 1\right), 1\right)$. Under the parameterized version of the model, $\left(l V^{\prime \prime} / V^{\prime}-l F_{l l} / F_{l}\right)=\sigma+\alpha$ and $k F_{l k} / F_{l}=\alpha$ holds, so that (44) simplifies to (42). In this equation the $\dot{k} / k$ term can be interpreted as follows: since the marginal product of labor $F_{l}(k, l)$ is homogeneous of degree zero, a rise in the growth of capital $\dot{k} / k$ results in a one-for-one increase in the growth rate of labor demand for a given growth rate of wages. In equilibrium, the growth rate of employment rises by less than one-for-one, since a rise in the growth rate of wages - generating an increase in the growth rate of labor supply - is required to maintain equilibrium in the labor market over time. ${ }^{7}$

In order to obtain a differential equation for equilibrium employment in the form $i=i(k, l)$, we must substitute for $\dot{k} / k$ by using (41). This yields

$$
i / l=\frac{1}{\alpha+\sigma}\left[-(\alpha+\beta)\left(\frac{(1-\alpha) B}{\mu(1+\sigma)}\right)^{\frac{1}{\theta}} k^{\frac{\alpha-\theta}{\theta}} l^{-\frac{\alpha+\sigma}{\theta}}+\rho\right] .
$$

With respect to the $i=0$ isocline given by

$$
\left.l\right|_{i=0}=\left(\frac{\alpha+\beta}{\rho}\right)^{\frac{\theta}{\alpha+\sigma}}\left(\frac{(1-\alpha) B}{\mu(1+\sigma)}\right)^{\frac{1}{\alpha+\sigma}} k^{\frac{\alpha-\theta}{\alpha+\sigma}}
$$

for $l>0$, we can distinguish three cases: i) if $\theta=\alpha$, then it is a horizontal line in the $(k, l)$ plane; ii) if $\theta>\alpha$, then it is negatively sloped and strictly convex; and iii) if $\theta<\alpha$, then it is positively sloped and strictly concave.

Observe that in all three cases a rise in $\beta$ causes the $i=0$ isocline to shift upwards. Because $\partial(i / l) / \partial l>0$, equilibrium employment is rising, $i>0$, above the $i=0$ isocline. More specifically, an increase in employment has the following effects. First, it impacts the effective rate of return $r^{e}$ which, according to the agent's Euler equation for work effort (43), changes the growth rate of labor supply. Observe that the reaction of $r^{e}$ to a rise in $l$ is ambiguous, because, on the one hand, the market rate of return $r=F_{k}$ increases, while, on the other hand, the status-dependent component, which equals $\beta c(k, l) k^{-1}$, declines.

\footnotetext{
${ }^{7}$ More specifically, a rise in $\dot{w} / w$ by one percentage point causes the growth rate of labor supply to rise by $\sigma^{-1}$ percentage points and the growth rate of labor demand to fall by $\alpha^{-1}$ percentage points.
} 
The first effect will tend to reduce the growth rate of labor supply, while the second effect will work in the opposite direction. There is an additional effect that guarantees a positive relationship between the growth rate of equilibrium employment, $i / l$, and $l$, as is evident from (45). Since $\partial \dot{k} / \partial l>0$ holds due to (41), the rise in $l$ also causes the rate of capital accumulation $\dot{k} / k$ to increase, which, as discussed above in detail in the context of (44), leads to a rise in the growth rates of both equilibrium employment and the real wage.

Now we are in a position to consider the transitional dynamics in the $(k, l)$ plane. In doing so we combine analytical results with the intuition gained from phase diagram analysis. Using the fact that $\operatorname{sgn}\left(m_{21}^{l}\right)=-\operatorname{sgn}(\alpha-\theta), m_{22}^{l}>0$ (see appendix 5.3.3) and $\xi_{1}<0$, it follows from (36) that the sign of the slope of the stable arm in the $(k, l)$ space equals the sign of the difference $\alpha-\theta$. Hence, if $\theta<\alpha$, then work effort rises (i.e., leisure declines) along with capital and consumption, while the opposite is the case if $\theta>\alpha$, a result that holds in both the socially planned and the decentralized economies.

Using (38) for $x=l$, it can be shown that a rise in $\beta$ causes the stable arm in the $(k, l)$ plane i) to shift upwards, and ii) to become flatter. Observe that both results hold, irrespective of whether the stable arm is positively $(\theta<\alpha)$ or negatively $(\theta>\alpha)$ sloped. Hence, the unambiguously positive shift effect on $l(0)$ is either reinforced by a positive slope effect if $\theta<\alpha$, or dampened by a negative slope effect if $\theta>\alpha$ (see appendix 5.3.4).

In our subsequent graphical analysis (see Figures $2 \mathrm{a}$ and $2 \mathrm{~b}$ ), we will restrict our attention to two special cases. Figure 2a illustrates the case $\theta=\alpha$ in which the stable arm is horizontal and coincides with the $i=0$ isocline. As discussed above, a rise in the status parameter $\beta$ causes the $i=0$ line to shift upwards. These properties imply that employment is constant over time in both the decentralized and the socially planned economy and that agents work too much at any time $t$ due to status preference. Taking into account that the $\dot{k}=0$ isocline is positively sloped because of $\theta=\alpha<1$, Figure 2a confirms that agents also accumulate too much physical capital (see Proposition 1B).

Figure $2 \mathrm{~b}$ depicts the other special case $\theta=1$ in which the $\dot{k}=0$ isocline is horizontal. Since $\alpha<\theta=1$, both the $i=0$ locus and the stable arm are negatively sloped. A rise in $\beta$ results in an upward shift of the $i=0$ isocline. Since, however, $\dot{k}=0$ is flat, this does not affect the steady-state value of employment. As indicated above, there is positive shift effect on $l(0)$ resulting from the upward shift of the stable arm, and a negative slope effect because the downward-sloping stable arm becomes flatter, i.e., its slope in absolute value 
declines. Nevertheless, as long as $k_{0}$ is sufficiently close to $\tilde{k}$, the net effect of an increase in the status parameter $\beta$ on initial work effort is always positive. Hence, while the level of work effort in the decentralized economy is socially optimal in the long run, agents work too hard in the short run.

Extending the phase diagram analysis to the remaining cases, which correspond to $\theta<\alpha, \alpha<\theta<1$, and $\theta>1$, we can obtain the following general result: as long as $\theta<1$, the decentralized economy is characterized by excessive work effort in both the short run and the long run, compared to the socially planned economy. If, however, $\theta>1$, then agents work too hard in the short run, but too little in the long run. The latter result, for example, can be confirmed by taking into account that for $\theta>1$ both isoclines and the stable arm are negatively sloped and that the $\dot{l}=0$ isocline intersects the $\dot{k}=0$ isocline from above and shifts upwards for an increase in $\beta$.

Above we have studied the stable arms in the $(k, c)$ and the $(k, l)$ plane. Before we leave this part of the paper, we briefly summarize the co-movements of the consumptioncapital ratio $c / k$ and the capital-labor ratio $k / l$, respectively, with physical capital $k$. This will prove very useful in studying the dynamics of the effective rate of return $r^{e}$ and the optimal tax rate. Similar to employment, the behavior of the consumption-capital ratio $c / k$ depends on the sign of the difference $\alpha-\theta$. If $\theta<\alpha$, then $c / k$ and $k$ always move in the same direction, while the opposite is the case if $\theta>\alpha$. In the special case $\theta=\alpha$, $c / k$ remains constant as $k$ evolves. In contrast, there is no ambiguity with respect to the co-movements of the capital-labor ratio $k / l$ and physical capital $k$. These variables always move in the same direction, regardless of whether $\theta<\alpha, \theta=\alpha$, or $\theta>\alpha$ holds. $^{8}$

\subsubsection{Numerical Simulation of Transitional Dynamics}

Our phase diagram analysis investigated the co-movements of physical capital and, respectively, consumption and work effort. It also revealed how the steady states and initial values for the control variables in the decentralized economies differ from their socially optimal counterparts. In order to complete the picture of transitional dynamics we plot the time paths of employment and consumption, respectively, in Figures $3 \mathrm{a}-\mathrm{b}$ and 4 . To do

\footnotetext{
${ }^{8}$ If the analysis is carried out in the $(k, \eta)$ plane, where $\eta \equiv c / k$, then the corresponding Jacobian $\mathbf{M}^{\eta}$ exhibits the property that $\operatorname{sgn}\left(m_{21}^{\eta}\right)=-\operatorname{sgn}(\alpha-\theta)$ and $m_{22}^{\eta}>0$. Analogously, the Jacobian $\mathbf{M}^{\kappa}$, where $\kappa \equiv k / l$, corresponding to the phase diagram in the $(k, \kappa)$ plane is characterized by the fact that $m_{11}^{\kappa}>0$ and $m_{12}^{\kappa}<0$ (for complete details see appendix 5.4 and 5.5). Our statements then follow from (36).
} 
so, we use the decentralized and socially optimal solutions given by (36) and (37) and calculate the implied time paths of employment and consumption under the assumption that the initial capital stock is the same in both economies and equal to $50 \%$ of the socially optimal steady-state value. In all figures the following parameter values for the instantaneous preferences (25) and the Cobb-Douglas production function (26) are assigned:

$$
\alpha=0.36, \quad \beta=0.04, \quad \mu=1, \quad B=1, \quad \sigma=0.5, \quad \rho=0.04 .
$$

The capital share $\alpha$ is $36 \%$, the value of the rate of time preference $\rho$ is $4 \%$, while $\sigma=0.5$ implies an intertemporal elasticity of substitution for individual labor supply of 2.0. For simplicity, we assign values of unity to the technology and preference parameters $\mu$ and $B$. Regarding the status preference parameter $\beta$, we choose a value of 0.04 . From the preceding analysis it became clear that the preference parameter $\theta$ - corresponding to the inverse of the intertemporal elasticity of substitution for consumption - is crucial in determining the short and long-run behavior of employment. For this reason, we first examine the intertemporal time paths of employment before considering those of consumption.

Regarding the dynamics of employment, we restrict our attention to the case in which $\theta$ takes the following two values, $\theta=(0.25,2.0)$, and which are illustrated, respectively, in Figures $3 \mathrm{a}-\mathrm{b} .{ }^{9}$ We consider these cases, because they provide additional information that cannot be readily obtained from our previous analysis of the $(k, l)$ phase diagram. Note that in these graphical illustrations the "solid" curve represents the path of decentralized employment, while the "dashed" curve corresponds to its socially optimal counterpart, which is calculated letting $\beta=0.0$ in the decentralized solutions. For example, for the case illustrated in Figure 3a where $\theta=0.25<\alpha=0.36$, we observe that work effort is rising whether or not the economy is in a private or socially optimal equilibrium. Moreover, in Figure 3a the path of employment in the decentralized economy always lies above the corresponding optimal path. In other words, the paths of decentralized and socially optimal work effort do not intersect if $\theta=0.25$. This reflects the non-optimality of the decentralized solution and the fact that agents work "too hard" not only in the short run, but also in long-run equilibrium if $\theta<1$. Considering next the case, depicted in Figure $3 \mathrm{~b}$, in which $\theta=2.0$, we observe that steady-state private work effort is less than that chosen by the social planner and employment is declining over time. Nevertheless, as we

\footnotetext{
${ }^{9}$ The value of $\theta$ equal to 2.0 is closer to values estimated by empirical research, since it implies a intertemporal elasticity of substitution equal to 0.5 .
} 
have shown above, private employment at $t=0$ exceeds its socially optimal level. This, in turn, means that there must be a time $t=t^{*}$ in Figure $3 \mathrm{~b}$ for which the decentralized and socially optimal paths of employment "cross", i.e., a point in time in which the two paths intersect. ${ }^{10}$

Finally, we turn to the transitional dynamics of consumption, which is illustrated for the case $\theta=0.25$ in Figure 4. We depict only this single case, since we can show that the behavior of consumption over time is qualitatively the same whether or not $\theta$ is greater or less than unity. Consistent with our above results, we observe in Figure 4 that decentralized initial consumption $c(0)$ falls short of the initial value that would obtain in the socially optimal economy. Again, private agents consume "too little" at $t=0$ compared to the social optimum. As, however, we have indicated above, this is a necessary counterpart of the fact that status-conscious agents accumulate "too much" physical capital during the transition to steady state. Because, eventually, the latter ensures over-consumption regardless of the long-run behavior of employment, this means that there exists a time $t=t^{*}$ for which the decentralized and socially optimal paths of consumption "cross", so that subsequent to $t=t^{*}$ private agents begin to over-consume. One aspect that does, however, depend on the value of $\theta$ is the timing of the intersection of the paths of decentralized and socially optimal consumption. In particular, we can show that the point of intersection occurs earlier in the adjustment phase as $\theta$ becomes larger.

\subsection{Optimal Taxation: A Note}

As indicated above, the inefficiencies in the relative wealth approach result from the fact that the effective rate of return on wealth accumulation exceeds the socially optimal rate, given by the marginal product of physical capital, i.e., $r^{e} \equiv F_{k}+\left(u_{z} / u_{c}\right) k^{-1}>F_{k}$. In the following we will show that this distortion caused by relative wealth preferences can be completely removed by taxing capital income appropriately. If the government imposes a tax on capital income and returns lump-sum transfers, then the flow budget constraint of the representative household equals

$$
\dot{a}=(1-\tau) r a+w l-c+q,
$$

\footnotetext{
${ }^{10}$ This crossing-pattern occurs only if $\theta>1$. For case (not illustrated) in which $\alpha<\theta<1$ decentralized employment is declining and always greater than employment in the socially optimal economy.
} 
where $\tau$ and $q$ denote, respectively, the tax rate on capital income and lump-sum transfers. We assume that each individual not only takes the time path of $A$, but also the evolution of $\tau$ and $q$ as given. As before, we will restrict attention to symmetric macroeconomic equilibria, so that $a=A$, and make the added assumption that the government continuously runs a balanced budget, i.e., $\tau r a=\tau r k=q$. The following proposition regarding the optimal taxation of capital income can then be obtained for our general specification of preferences.

Proposition 2 If the government sets the tax rate $\tau$ on capital income according to the rule

$$
\tau=\Phi(C, L, K) \equiv \frac{u_{z}(C, L, 1) K^{-1}}{u_{c}(C, L, 1) F_{k}(K, L)},
$$

where $C, K$, and $L$ denote the average values of consumption, capital and hours worked, and rebates total tax revenues as lump-sum transfers, then the social optimum is attained in the decentralized economy with relative wealth preferences.

We wish to stress the following two aspects. First, since the optimal tax rate is a function of average values, $\tau=\Phi(C, L, K)$, each agent takes its time path, similar to those of the interest rate $r$ and the real wage $w$, as given. Second, because applying the optimal tax rule ensures that $x^{d}(t)=x^{p}(t)$ for $t \geq 0$, where $x$ denotes any variable of the model, the time path of the optimal tax rate satisfies $\tau(t)=\Phi\left(c^{p}(t), l^{p}(t), k^{p}(t)\right)$.

The idea of the proof of (46), the details of which are provided in appendix 5.6.1, is straightforward: the goal of the welfare-maximizing government is to impose a tax rule $\tau=\Phi(C, L, K)$ guaranteeing that in the symmetric equilibrium of the decentralized economy the after-tax effective rate of return "reproduces" the socially optimal rate, so that

$$
[1-\Phi(c, l, k)] F_{k}(k / l, 1)+\frac{u_{z}(c, l, 1) k^{-1}}{u_{c}(c, l, 1)}=F_{k}(k / l, 1)
$$

holds for all $c, l$, and $k$.

To gain additional insights regarding the behavior of the optimal tax, we employ the parameterized version of model, based on the illustration (25). Recall that the statusrelated component of the effective rate of return $r^{e}$ equals $\left[u_{z}(c, l, 1) / u_{c}(c, l, 1)\right] k^{-1}=$ $\beta(c / k)$. The goal of optimal tax policy is to "remove" the resulting distortion so that $x^{d}(t)=x^{p}(t)$ for $t \geq 0$, which requires, according to (47), that the time path of the 
optimal tax rate satisfies the following condition:

$$
\tau F_{k}\left(k^{p} / l^{p}, 1\right)=\beta\left(c^{p} / k^{p}\right) .
$$

Observe that the time path of the optimal tax rate depends on how the before-tax market rate of return and the status-related component evolve along the socially optimal time paths of the capital-labor ratio and the consumption-capital ratio. As we showed above, the capital-labor ratio $\kappa \equiv k / l$ moves in the same direction over time as physical capital, regardless of whether $\beta=0$ or $\beta>0$. This, in turn, implies that if $k_{0}<\tilde{k}$, then the beforetax market rate of return along the socially optimal path, $F_{k}\left(\kappa^{p}, 1\right)$, declines over time as $\kappa^{p}$ rises. In contrast, for $\beta \geq 0$ the co-movements of the consumption-capital ratio $(c / k)$ and physical capital $k$, as indicated at the end of subsubsection 3.2.2, are ambiguous and depend on the sign of the difference $\alpha-\theta$. Consider, first, the special case $\theta=\alpha$. Here, the consumption-capital ratio - and, thus, the status-dependent component evaluated along the socially optimal path, $\beta\left(c^{p} / k^{p}\right)$-is not changing over time. Consequently, the optimal $\operatorname{tax} \tau$ rises as $F_{k}\left(\kappa^{p}, 1\right)$ falls to maintain (48) and exactly offset the constant distortion represented by $\beta\left(c^{p} / k^{p}\right)$. If $\theta<\alpha$, then the ratio $\left(c^{p} / k^{p}\right)$ does not remain constant, but increases as $k^{p}$ increases. It is obvious, then, that the optimal $\tau$ must increase over time to ensure the condition (48) and offset the rising relative wealth distortion. Even if $\theta>\alpha-$ the case in which the ratio $\left(c^{p} / k^{p}\right)$ falls over time as $k^{p}$ rises-we can show analytically that $\tau$ must also rise, which is attributable to the fact that $F_{k}\left(\kappa^{p}, 1\right)$ declines more rapidly than $\beta\left(c^{p} / k^{p}\right)$.

In order to convey the idea of the proof, we substitute $F_{k}(k, l)=\alpha B(k / l)^{-(1-\alpha)}$ into (48) and solve for $\tau$ :

$$
\tau=\frac{\beta\left(c^{p} / k^{p}\right)}{F_{k}\left(k^{p} / l^{p}, 1\right)}=\frac{\beta}{\alpha B} c^{p}\left(k^{p}\right)^{-\alpha}\left(l^{p}\right)^{-(1-\alpha)}=\frac{\beta}{\alpha} \frac{c^{p}}{y^{p}},
$$

where $y^{p}=B\left(k^{p}\right)^{\alpha}\left(l^{p}\right)^{1-\alpha}$. First, note that the optimal tax rate converges to $\tilde{\tau} \equiv \beta / \alpha$ in the long run, because, due to the simplifying assumptions made in our model, steadystate consumption equals steady-state output, $\tilde{c}=\tilde{y} .{ }^{11}$ Linearizing (49) around the steady state, we derive in the appendix 5.6.2 the following expression that governs the dynamic

\footnotetext{
${ }^{11}$ Observe that $\tilde{\tau}$ is independent of $\theta^{-1}$, the elasticity of intertemporal substitution for consumption. Further, the numerical parameterization used above yields a value of $11 \%$ for the steady-state optimal tax. Note that tripling the status parameter $\beta$ to a (feasible) value of 0.12 yields a $\tilde{\tau}$ of $33 \%$, which is close to real-world values.
} 
evolution of the optimal tax rate:

$$
\tau(t)=\frac{\beta}{\alpha}+\frac{-\xi_{1}^{p}}{\phi}\left(k^{p}(t)-\tilde{k}^{p}\right), \quad k^{p}(t)-\tilde{k}^{p}=\left(k_{0}-\tilde{k}^{p}\right) e^{\xi_{1}^{p} t}
$$

where $\xi_{1}^{p}=\left.\xi_{1}\right|_{\beta=0}$ and $\phi$ is a positive constant. Since $\xi_{1}^{p}<0$ and $\phi>0$, the optimal tax rate and physical capital always move in the same direction in our parameterized model.

Furthermore, according to $\tilde{\tau} \equiv \beta / \alpha$, there exists a one-to-one relationship between the optimal tax rate $\tau$ and the status parameter $\beta$ in the long-run. From (48), $\tau F_{k}\left(k^{p} / l^{p}, 1\right)=$ $\beta\left(c^{p} / k^{p}\right)$, and the fact that the time paths of $x^{p}$ are independent of $\beta$ in our model, it follows that this relationship holds for all $t \geq 0$.

Using our numerical model, it is straightforward to depict the rising adjustment paths of $\tau$, although we do not illustrate them here. Letting $\theta=(0.25,2.0)$, we can show that the optimal tax at $t=0$ is lower for $\theta=0.25$ than it is for $\theta=2.0$. This reflects the fact that a smaller value of $\theta$, i.e., a greater willingness to substitute consumption over time, leads to a lower initial level of consumption, $c^{p}(0)$, which, in turn, implies that the status-dependent component of the effective rate of return, $\beta\left(c^{p} / k^{p}\right)$, is less important and, consequently, requires a smaller initial optimal tax.

\section{Conclusion}

We examine in this paper the effects of the quest for status within a Ramsey-type model in which labor supply is endogenously determined and agents are homogeneous. Although in our general model we allow the agent's status to be determined either by her relative consumption or her relative wealth, our analysis focuses on the latter case. In general, status preferences lead to inefficient outcomes due to externalities. In the relative consumption framework the willingness to substitute consumption for leisure is too high, while in the relative wealth approach the effective rate of return that consists of the market interest rate and a status-related component exceeds the socially optimal level.

Our analysis divides into two parts: a general treatment in which we consider the longrun implications of status preferences and a specific illustration that permits us to study the saddle path dynamics of the control variables consumption and work effort in the relative wealth framework. Under both specifications of the quest for status, the steady state possesses the property that consumption and the stock of physical capital are greater than their socially optimal counterparts. While in the relative consumption approach the 
steady state is always characterized by excessive work effort, there are specifications of relative wealth preferences in which agents work too little in the long run. In the latter case agents, nevertheless, can "afford" excessive consumption, since, in contrast to the relative consumption approach, the capital-labor ratio and the output per hours worked exceed their socially optimal levels. Moreover, although agents in this case consume both more leisure and more goods in the long run than in the socially planned economy, they are worse off as measured by intertemporal utility. This is due to the fact that the excessive capital accumulation is achieved by under-consumption and excessive work effort in the initial phase of the planning horizon. While the results for the steady state hold for quite general specifications of relative wealth preferences, a detailed analysis of transitional dynamics requires the introduction of simplifying assumptions on the utility function. In the phase diagram analysis and the numerical simulations we specify that work effort is additively separable from own consumption and status. Along the stable arm, consumption always moves in the same direction as physical capital. In contrast, the co-movement of work effort with physical capital is ambiguous: if the elasticity of intertemporal substitution of consumption is less than the inverse of the capital share, then work effort decreases as physical capital increases. In the opposite case in which the willingness to substitute consumption over time is relatively high, the results are reversed. These qualitative results with respect to transitional dynamics hold for both the decentralized economy and the socially planned one.

In the decentralized economy there is both under-consumption and excessive work effort in the initial phase of transitional dynamics, where this deviation from the socially optimal solution is amplified by a rise in the degree of status consciousness. In the empirically plausible case in which the elasticity of intertemporal substitution of consumption is less than unity, agents work too little in the long run. Hence, the time paths of decentralized and socially optimal work effort intersect, i.e., there is too much work effort in the short run and too little in the long run. In contrast to employment, the time paths of decentralized and socially optimal consumption intersect for all admissible parameter values, i.e., there is always under-consumption in the short run and excessive consumption in the long run. In addition, we demonstrate that an increase in the status parameter "slows down" the economy's speed of convergence along stable saddle path.

Finally, we show that the social optimum can be replicated in the decentralized econ- 
omy by optimally taxing capital income, where the optimal tax rate depends positively on the degree of status consciousness and increases as physical capital accumulates. Optimal taxation ensures that the after-tax effective rate of return "reproduces" the one in the socially optimal economy.

\section{Appendix}

\subsection{The steady-state values}

The steady-state values of $c, l, k$, and $\kappa$ are given by

$$
\begin{gathered}
\tilde{c}=\left(\frac{(1-\alpha)}{\mu(1+\sigma)} B^{\frac{1+\sigma}{1-\alpha}}\left(\frac{\alpha+\beta}{\rho}\right)^{\frac{\alpha(1+\sigma)}{1-\alpha}}\right)^{\frac{1}{\sigma+\theta}}, \\
\tilde{l}=\left(\frac{(1-\alpha)}{\mu(1+\sigma)} B^{\frac{1-\theta}{1-\alpha}}\left(\frac{\alpha+\beta}{\rho}\right)^{\frac{\alpha(1-\theta)}{1-\alpha}}\right)^{\frac{1}{\sigma+\theta}}, \\
\tilde{k}=\left(\frac{(1-\alpha)}{\mu(1+\sigma)} B^{\frac{1+\sigma}{1-\alpha}}\left(\frac{\alpha+\beta}{\rho}\right)^{\frac{\alpha+\sigma+\theta(1-\alpha)}{1-\alpha}}\right)^{\frac{1}{\sigma+\theta}}, \\
\tilde{\kappa}=\left(\frac{(\alpha+\beta) B}{\rho}\right)^{\frac{1}{1-\alpha}} .
\end{gathered}
$$

\subsection{Control-State Space Analysis in the $(k, c)$ plane}

\subsubsection{The Jacobi matrix $\mathrm{M}^{c}$}

In order to derive a system of differential equations in $k$ and $c$, the necessary optimality conditions (28) and (29) are first solved for $l$ and $\lambda$ in the following form:

$$
l=\left(\frac{(1-\alpha) B}{\mu(1+\sigma)}\right)^{\frac{1}{\alpha+\sigma}} k^{\frac{\alpha}{\alpha+\sigma}} c^{-\frac{\theta}{\alpha+\sigma}}, \quad \lambda=c^{-\theta} .
$$

Substitution of these results into (27) and (30) then yields the following differential equations:

$$
\begin{gathered}
\dot{k}=\left(\frac{1-\alpha}{\mu(1+\sigma)}\right)^{\frac{1-\alpha}{\alpha+\sigma}} B^{\frac{1+\sigma}{\alpha+\sigma}} k^{\frac{\alpha(1+\sigma)}{\alpha+\sigma}} c^{-\frac{\theta(1-\alpha)}{\alpha+\sigma}}-c \\
\dot{c}=\frac{c}{\theta}\left[\alpha\left(\frac{1-\alpha}{\mu(1+\sigma)}\right)^{\frac{1-\alpha}{\alpha+\sigma}} B^{\frac{1+\sigma}{\alpha+\sigma}} k^{-\frac{(1-\alpha) \sigma}{\alpha+\sigma}} c^{-\frac{(1-\alpha) \theta}{\alpha+\sigma}}+\beta(c / k)-\rho\right] .
\end{gathered}
$$

The steady-state values $\tilde{k}$ and $\tilde{c}$, which are determined by setting $\dot{k}=0$ and $\dot{c}=0$, are given by (52) and (50). Differentiating $\dot{k}(k, c)$ and $\dot{c}(k, c)$ with respect to $k$ and $c$ and 
evaluating the resulting expressions at $(\tilde{k}, \tilde{c})$ yields the Jacobi matrix $\mathbf{M}^{c}$. Its elements are given by

$$
\begin{gathered}
m_{11}^{c}=\frac{\alpha(1+\sigma) \rho}{(\alpha+\beta)(\alpha+\sigma)}, \quad m_{12}^{c}=-\frac{\theta(1-\alpha)+\alpha+\sigma}{\alpha+\sigma} \\
m_{21}^{c}=-\frac{\rho^{2}}{\theta(\alpha+\beta)^{2}}\left(\frac{\alpha(1-\alpha) \sigma}{\alpha+\sigma}+\beta\right), \quad m_{22}^{c}=-\frac{\rho}{(\alpha+\beta) \theta}\left(\frac{\alpha(1-\alpha) \theta}{\alpha+\sigma}-\beta\right) .
\end{gathered}
$$

\subsubsection{Shift and Slope effects of changes in $\beta$ in the $(k, c)$ plane}

First, we will show that $\partial \xi_{1} / \partial \beta>0$, and thus $\partial\left|\xi_{1}\right| / \partial \beta<0$ holds for all $\theta>0$. Implicit differentiation of the characteristic equation (32) yields

$$
\frac{\partial \xi_{1}}{\partial \beta}=\frac{\rho\left[(1-\alpha)(\sigma+\theta) \rho-\alpha(1-\theta)(\alpha+\sigma) \xi_{1}\right]}{(\alpha+\beta)(\alpha+\sigma)\left[(\alpha \theta+\beta) \rho-2(\alpha+\beta) \theta \xi_{1}\right]} .
$$

Note that $\theta<1$ is sufficient for $\partial \xi_{1} / \partial \beta>0$, which, in turn, implies that $\partial\left|\xi_{1}\right| / \partial \beta<0$. In case that $\theta>1$ we have

$$
\frac{\partial \xi_{1}}{\partial \beta}>0 \Longleftrightarrow-\frac{(1-\alpha)(\sigma+\theta) \rho}{(\theta-1) \alpha(\alpha+\sigma)}<\xi_{1} \Longleftrightarrow P\left(-\frac{(1-\alpha)(\sigma+\theta) \rho}{(\alpha+\sigma) \alpha(\theta-1)}\right)>0,
$$

where the second equivalence is easily verified by using a graphical representation of the strictly convex characteristic polynomial and taking into account that $P\left(\xi_{1}\right)=0$ and $P(\xi)>0$ for $\xi<\xi_{1}$. Since

$$
\begin{aligned}
& P\left(-\frac{(1-\alpha)(\sigma+\theta) \rho}{(\alpha+\sigma) \alpha(\theta-1)}\right) \\
= & \frac{(1-\alpha)[(\theta-1)+(1-\alpha)](\sigma+\theta)[(\sigma+\alpha)+\theta(1-\alpha)](\alpha+\beta) \rho^{2}}{\theta \alpha^{2}(\alpha+\beta)(\alpha+\sigma)^{2}(\theta-1)^{2}}>0,
\end{aligned}
$$

we obtain that $\partial \xi_{1} / \partial \beta>0$, and thus $\partial\left|\xi_{1}\right| / \partial \beta<0$ holds for all $\theta>0$.

Second, we evaluate (38) for the case in which $x=c$ and obtain

$$
\begin{gathered}
\frac{\partial \tilde{c}}{\partial \beta}-\frac{m_{11}^{c}-\xi_{1}}{-m_{12}^{c}} \frac{\partial \tilde{k}}{\partial \beta}=\frac{(\alpha+\sigma) \xi_{1}}{(1-\alpha)(\alpha+\beta)(\sigma+\theta)} \tilde{k}<0, \\
\frac{\partial\left[\left(m_{11}^{c}-\xi_{1}\right) /\left(-m_{12}^{c}\right)\right]}{\partial \beta}=-\frac{\alpha+\sigma}{\theta(1-\alpha)+\alpha+\sigma}\left(\frac{\alpha(1+\sigma)}{(\alpha+\beta)^{2}(\alpha+\sigma)}+\frac{\partial \xi_{1}}{\partial \beta}\right)<0 .
\end{gathered}
$$

Because $\xi_{1}<0$, the first result implies that a rise in $\beta$ causes the stable arm in the $(k, c)$ plane to shift downwards. Taking into account that $\partial \xi_{1} / \partial \beta>0$ holds, as demonstrated above, the second result shows that the stable saddle path becomes flatter as $\beta$ increases. 


\subsection{Control-State Space Analysis in the $(k, l)$ plane}

\subsubsection{The Euler Equation for Individual Labor Supply (43)}

If the utility function $u(c, l, z)$ takes the general additively separable form $U(c, z)+V(l)$, then the necessary optimality conditions (10)-(11) and the differential equation for the costate variable (12) can be written as follows:

$$
\begin{gathered}
U_{c}(c, a / A)-\lambda=0, \\
V^{\prime}(l)+\lambda w=0, \\
\dot{\lambda} / \lambda=-\left[r+\frac{U_{z}(c, a / A) A^{-1}}{U_{c}(c, a / A)}-\rho\right] .
\end{gathered}
$$

Equations (55) and (56) can can be solved for $c$ and $\lambda$ in the following form:

$$
c=\hat{c}(w, l, a / A), \quad \lambda=\hat{\lambda}(w, l) \equiv-V^{\prime}(l) / w
$$

Differentiating $\lambda=\hat{\lambda}(w, l)$ with respect to time $t$ and and dividing the resulting expression by $\lambda$ we obtain

$$
\frac{\dot{\lambda}}{\lambda}=\frac{l V^{\prime \prime}(l)}{V^{\prime}(l)} \frac{\dot{l}}{l}-\frac{\dot{w}}{w}
$$

Substituting (58) into (57) and rearranging yields

$$
\bar{l}=\frac{V^{\prime}(l)}{l V^{\prime \prime}(l)}\left\{\frac{\dot{w}}{w}-\left[r+\frac{U_{z}(\hat{c}(w, l, a / A), a / A) A^{-1}}{U_{c}(\hat{c}(w, l, a / A), a / A)}\right]+\rho\right\}
$$

which is equivalent to (43).

\subsubsection{The Euler Equation for Equilibrium Employment (44)}

Due to the assumption of perfect foresight each agent knows that the equilibrium levels of the rental rate of capital and the real wage satisfy $r=F_{k}(K, L)$ and $w=F_{l}(K, L)$, where $K$ and $L$ denote the average physical capital and average hours worked in the economy. Observe that since each agent takes the time paths of $K$ and $L$ as given, she also takes the time path of $r$ and $w$ as given. From $w=F_{l}(K, L)$ it then follows that

$$
\frac{\dot{w}}{w}=\frac{K F_{l k}(K, L)}{F_{l}(K, L)} \frac{\dot{K}}{K}+\frac{L F_{l l}(K, L)}{F_{l}(K, L)} \frac{\dot{L}}{L}
$$


Substitution of $r=F_{k}(K, L)$ and (60) into (59) shows that the agent's Euler equation for labor supply can be rewritten as

$$
\begin{aligned}
\frac{i}{l}=\frac{V^{\prime}(l)}{l V^{\prime \prime}(l)}\{ & \frac{K F_{l k}(K, L)}{F_{l}(K, L)} \frac{\dot{K}}{K}+\frac{L F_{l l}(K, L)}{F_{l}(K, L)} \frac{\dot{L}}{L} \\
& \left.-\left[F_{k}(K, L)+\frac{U_{z}\left(\hat{c}\left(F_{l}(K, L), l, a / A\right), a / A\right) A^{-1}}{U_{c}\left(\hat{c}\left(F_{l}(K, L), l, a / A\right), a / A\right)}\right]+\rho\right\} .
\end{aligned}
$$

In a symmetric macroeconomic equilibrium $l=L$ and $a=A=K=k$ holds. Substituting these relations into the agent's Euler equation and rearranging we obtain the following Euler equation for equilibrium employment:

$$
\begin{aligned}
\frac{i}{l}=\left[\frac{l V^{\prime \prime}(l)}{V^{\prime}(l)}-\frac{l F_{l l}(k, l)}{F_{l}(k, l)}\right]^{-1}\left\{\frac{k F_{l k}(k, l)}{F_{l}(k, l)} \frac{\dot{k}}{k}\right. \\
\left.-\left[F_{k}(k, l)+\frac{U_{z}\left(\hat{c}\left(F_{l}(k, l), l, 1\right), 1\right) k^{-1}}{U_{c}\left(\hat{c}\left(F_{l}(k, l), l, 1\right), 1\right)}\right]+\rho\right\} .
\end{aligned}
$$

This representation is equivalent to (44).

\subsubsection{The Jacobi matrix $\mathrm{M}^{l}$}

In order to derive a system of differential equations in $k$ and $l$, the necessary optimality conditions (28) and (29) can be solved for $c$ and $\lambda$ in the following form:

$$
c=\left(\frac{(1-\alpha) B}{\mu(1+\sigma)}\right)^{\frac{1}{\theta}} k^{\frac{\alpha}{\theta}} l^{-\frac{\alpha+\sigma}{\theta}}, \quad \lambda=\left(\frac{(1-\alpha) B}{\mu(1+\sigma)}\right)^{-1} k^{-\alpha} l^{\alpha+\sigma} .
$$

The latter equation implies that $\dot{\lambda} / \lambda=-\alpha(\dot{k} / k)+(\alpha+\sigma)(i / l)$. Substitution of these results into (27) and (30) yields the following system of differential equations:

$$
\begin{gathered}
\dot{k}=B k^{\alpha} l^{1-\alpha}-\left(\frac{(1-\alpha) B}{\mu(1+\sigma)}\right)^{\frac{1}{\theta}} k^{\frac{\alpha}{\theta}} l^{-\frac{\alpha+\sigma}{\theta}}, \\
\dot{l}=-\frac{l}{\alpha+\sigma}\left[(\alpha+\beta)\left(\frac{(1-\alpha) B}{\mu(1+\sigma)}\right)^{\frac{1}{\theta}} k^{\frac{\alpha-\theta}{\theta}} l^{-\frac{\alpha+\sigma}{\theta}}-\rho\right] .
\end{gathered}
$$

Linearization about the steady state yields the Jacobi matrix $\mathbf{M}^{l}$. Its elements are given by

$$
\begin{gathered}
m_{11}^{l}=-\frac{\alpha \rho(1-\theta)}{\theta(\alpha+\beta)}, \quad m_{12}^{l}=\left(1-\alpha+\frac{\alpha+\sigma}{\theta}\right) B^{\frac{1}{1-\alpha}}\left(\frac{\alpha+\beta}{\rho}\right)^{\frac{\alpha}{1-\alpha}}, \\
m_{21}^{l}=-\frac{(\alpha-\theta)(\alpha+\beta)}{(\alpha+\sigma) \theta} B^{-\frac{1}{1-\alpha}}\left(\frac{\alpha+\beta}{\rho}\right)^{-\frac{2-\alpha}{1-\alpha}}, \quad m_{22}^{l}=\frac{\rho}{\theta} .
\end{gathered}
$$




\subsubsection{Shift and slope effect of changes in $\beta$ in the $(k, l)$ plane}

Evaluating (38) for $x=l$ we find that

$$
\begin{aligned}
& \frac{\partial \tilde{l}}{\partial \beta}-\frac{m_{11}^{l}-\xi_{1}}{-m_{12}^{l}} \frac{\partial \tilde{k}}{\partial \beta}=-\frac{\xi_{1} \theta \tilde{k}}{(1-\alpha)(\alpha+\beta)(\sigma+\theta)} B^{-\frac{1}{1-\alpha}}\left(\frac{\alpha+\beta}{\rho}\right)^{-\frac{\alpha}{1-\alpha}}>0, \\
& \frac{\partial\left[\left(-m_{21}^{l}\right) /\left(m_{22}^{l}-\xi_{1}\right)\right]}{\partial \beta}=m_{21}^{l} \frac{\left[(\alpha \theta+\beta) \rho-(\alpha+\beta) \theta(1+\alpha) \xi_{1}\right]}{(1-\alpha)(\alpha+\beta) \theta\left(-\xi_{1}\right)\left(m_{22}^{l}-\xi_{1}\right)^{2}} \frac{\partial \xi_{1}}{\partial \beta} .
\end{aligned}
$$

Using the fact that $\xi_{1}<0, \partial \xi_{1} / \partial \beta>0$ and the findings given in appendix 5.3.3, $\operatorname{sgn}\left(m_{21}^{l}\right)=-\operatorname{sgn}(\alpha-\theta)$, and $m_{22}^{l}>0$, it is clear that

$$
\operatorname{sgn}\left(\frac{\partial\left[\left(-m_{21}^{l}\right) /\left(m_{22}^{l}-\xi_{1}\right)\right]}{\partial \beta}\right)=\operatorname{sgn}\left(m_{21}^{l}\right)=-\operatorname{sgn}\left(\frac{-m_{21}^{l}}{m_{22}^{l}-\xi_{1}}\right)=-\operatorname{sgn}(\alpha-\theta) .
$$

Hence, a rise in $\beta$ causes the stable arm in the $(k, l)$ plane i) to shift upwards, and ii) to become flatter, i.e., the absolute value of its slope falls. Observe that both results hold, irrespective of whether the stable arm is positively $(\theta<\alpha)$ or negatively $(\theta>\alpha)$ sloped.

\subsection{Control-State Space Analysis in the $(k, c / k)$ plane}

In the additional appendix (not intended for publication) we demonstrate that the dynamic evolution of $k$ and $\eta \equiv c / k$ is governed by the following system of differential equations:

$$
\begin{gathered}
\dot{k}=B\left(\frac{(1-\alpha) B}{\mu(1+\sigma)}\right)^{\frac{1-\alpha}{\alpha+\sigma}} k^{\frac{\alpha(1+\sigma)-\theta(1-\alpha)}{\alpha+\sigma}} \eta^{-\frac{(1-\alpha) \theta}{\alpha+\sigma}}-\eta k, \\
\dot{\eta}=\frac{\eta}{\theta}\left[(\alpha-\theta) B\left(\frac{(1-\alpha) B}{\mu(1+\sigma)}\right)^{\frac{1-\alpha}{\alpha+\sigma}} k^{-\frac{(1-\alpha)(\sigma+\theta)}{\alpha+\sigma}} \eta^{-\frac{(1-\alpha) \theta}{\alpha+\sigma}}+(\beta+\theta) \eta-\rho\right] .
\end{gathered}
$$

The steady-state values $\tilde{k}$ and $\tilde{\eta}$ are determined by setting $\dot{k}=0$ and $\dot{\eta}=0$. It is easily verified that the solution for $\tilde{k}$ equals (52), while $\tilde{\eta}=\rho(\alpha+\beta)^{-1}$. Differentiating $\dot{k}(k, \eta)$ and $\dot{\eta}(k, \eta)$ with respect to $k$ and $\eta$ and evaluating the resulting expressions at $(\tilde{k}, \tilde{\eta})$ in order to obtain the elements of the Jacobian $\mathbf{M}^{\eta}$ we can show that

$$
\begin{gathered}
m_{11}^{\eta}=-\frac{(1-\alpha) \rho(\sigma+\theta)}{(\alpha+\sigma)(\alpha+\beta)} \\
m_{12}^{\eta}=-\frac{\alpha+\sigma+(1-\alpha) \theta}{\alpha+\sigma} B^{\frac{\alpha+\sigma}{(\sigma+\theta)(1-\alpha)}}\left(\frac{(1-\alpha) B}{\mu(1+\sigma)}\right)^{\frac{1}{\sigma+\theta}}\left(\frac{\alpha+\beta}{\rho}\right)^{\frac{\alpha+\sigma+(1-\alpha) \theta}{(1-\alpha)(\sigma+\theta)}}
\end{gathered}
$$




$$
\begin{gathered}
m_{21}^{\eta}=-\frac{(\alpha-\theta)(1-\alpha)(\sigma+\theta)}{\theta(\alpha+\sigma)}\left(\frac{(1-\alpha) B}{\mu(1+\sigma)}\right)^{-\frac{1}{\sigma+\theta}} \times \\
\times B^{-\frac{\alpha+\sigma}{(1-\alpha)(\sigma+\theta)}\left(\frac{\alpha+\beta}{\rho}\right)^{-\frac{(\alpha+\sigma+\theta(1-\alpha))+(1-\alpha)(\sigma+\theta)+(\sigma+\theta)(1-\alpha)}{(\sigma+\theta)(1-\alpha)}},} \\
m_{22}^{\eta}=\frac{\left[(1-\alpha) \theta^{2}+\left(\sigma+\alpha^{2}\right) \theta+(\alpha+\sigma) \beta\right] \rho}{\theta(\alpha+\beta)(\alpha+\sigma)}>0 .
\end{gathered}
$$

From $\operatorname{sgn}\left[-m_{21}^{\eta} /\left(m_{22}^{\eta}-\xi_{1}\right)\right]=\operatorname{sgn}(\alpha-\theta)$, it then follows that the sign of the slope of the stable arm in the $(k, c / k)$ plane equals the sign of $(\alpha-\theta)$.

\subsection{Control-State Space Analysis in the $(k, k / l)$ plane}

In the additional appendix (not for publication) we show that the dynamic evolution of $k$ and $\kappa=k / l$ is governed by the following system of differential equations:

$$
\begin{gathered}
\dot{k}=B k \kappa^{-(1-\alpha)}-\left(\frac{(1-\alpha) B}{\mu(1+\sigma)}\right)^{\frac{1}{\theta}} k^{-\frac{\sigma}{\theta}} \kappa^{\frac{\alpha+\sigma}{\theta}} \\
\dot{\kappa}=\frac{\kappa}{\alpha+\sigma}\left[(\alpha+\sigma) B \kappa^{-(1-\alpha)}+(\beta-\sigma)\left(\frac{(1-\alpha) B}{\mu(1+\sigma)}\right)^{\frac{1}{\theta}} k^{-\frac{\sigma+\theta}{\theta}} \kappa^{\frac{\alpha+\sigma}{\theta}}-\rho\right] .
\end{gathered}
$$

The steady-state values $\tilde{k}$ and $\tilde{\kappa}$ are given by (52) and (53). Differentiating $\dot{k}(k, \kappa)$ and $\dot{\kappa}(k, \kappa)$ with respect to $k$ and $\kappa$ and evaluating the resulting expression at the steady state $(\tilde{k}, \tilde{\kappa})$ in order to calculate the elements of the of the Jacobian $\mathbf{M}^{\kappa}$ we can show that

$$
\begin{gathered}
m_{11}^{\kappa}=\frac{(\theta+\sigma) \rho}{(\alpha+\beta) \theta}>0 \\
m_{12}^{\kappa}=-\frac{(\alpha+\sigma)+(1-\alpha) \theta}{\theta} B^{\frac{\alpha-\theta}{(1-\alpha)(\sigma+\theta)}\left(\frac{(1-\alpha) B}{\mu(1+\sigma)}\right)^{\frac{1}{\sigma+\theta}}\left(\frac{\alpha+\beta}{\rho}\right)^{\frac{\alpha-\theta-(1-\alpha) \sigma}{(1-\alpha)(\sigma+\theta)}}<0 .}
\end{gathered}
$$

Hence, the slope of the stable arm in the $(k, k / l)$ plane, which, according to (36), is expressed as $\left(m_{11}^{\kappa}-\xi_{1}\right) /\left(-m_{12}^{\kappa}\right)$, is unambiguously positive.

\subsection{Optimal Taxation}

\subsubsection{Proof of Proposition 2}

If the government imposes a tax on capital income and runs a balanced budget for all $t$ by returning lump-sum transfers, then the costate equation (14) is replaced by

$$
\dot{\lambda}=-\left[(1-\tau) F_{k}(k, l)+\frac{u_{z}(c, l, 1) k^{-1}}{u_{c}(c, l, 1)}-\rho\right] \lambda
$$


while all other equations that determine the decentralized solution remain unchanged and coincide with their socially optimal counterparts. Since the representative individual takes the time paths of $C, L$, and $K$ as given, she also takes the time path of the tax rate $\tau=\Phi(C, L, K)$ as given. In a symmetric equilibrium in which identical individuals make identical choices, $\tau=\Phi(c, l, k)$ holds. According to (46) we have

$$
\tau=\Phi(c, l, k)=\frac{u_{z}(c, l, 1) k^{-1}}{u_{c}(c, l, 1) F_{k}(k, l)} .
$$

Substitution of this result into $(65)$ yields $\dot{\lambda}=-\left[F_{k}(k, l)-\rho\right] \lambda$, which coincides with the differential equation (8) that governs the dynamic evolution of the shadow value $\lambda$ in the socially planned economy. Hence, the tax policy given in Proposition 2 implies that the dynamic evolution of the variables in the decentralized economy and the socially planned economy are governed by identical sets of equations and conditions. Therefore, the decentralized economy under the tax rule (46) replicates the socially planned one.

\subsubsection{The optimal tax rate}

First, the optimal tax rate given (49) can be rewritten as

$$
\tau=\frac{\beta}{\alpha B} \eta^{p}\left(\kappa^{p}\right)^{1-\alpha}
$$

where $\eta=c / k$ and $\kappa=k / l$. Linearization around the steady state $\left(\tilde{\eta}^{p}, \tilde{\kappa}^{p}\right)$ yields

$$
\tau=\tilde{\tau}+\frac{\beta}{\alpha B}\left(\tilde{\kappa}^{p}\right)^{1-\alpha}\left(\eta^{p}-\tilde{\eta}^{p}\right)+(1-\alpha) \frac{\beta}{\alpha B}\left(\tilde{\eta}^{p}\right)\left(\tilde{\kappa}^{p}\right)^{-\alpha}\left(\kappa^{p}-\tilde{\kappa}^{p}\right) .
$$

The steady-state values $\tilde{\tau}, \tilde{\eta}^{p}$ and $\tilde{\kappa}^{p}$ are obtained by using (50), (52) and (53) and setting $\beta=0$ :

$$
\tilde{\tau}=\beta / \alpha, \quad \tilde{\eta}^{p}=\rho / \alpha, \quad \tilde{\kappa}^{p}=(\alpha B / \rho)^{\frac{1}{1-\alpha}} .
$$

From (36) it follows that

$$
\eta^{p}-\tilde{\eta}^{p}=\frac{m_{11}^{\eta, p}-\xi_{1}^{p}}{-m_{12}^{\eta, p}}\left(k^{p}(t)-\tilde{k}^{p}\right), \quad \kappa^{p}-\tilde{\kappa}^{p}=\frac{m_{11}^{\kappa, p}-\xi_{1}^{p}}{-m_{12}^{\kappa, p}}\left(k^{p}(t)-\tilde{k}^{p}\right),
$$

where $\xi_{1}^{p}=\left.\xi_{1}\right|_{\beta=0}$ and $m_{i j}^{x, p}=\left.m_{i j}^{x}\right|_{\beta=0}$. Substituting (67) and (68) into (66) and using the expressions for $m_{i j}^{\eta, p}$ and $m_{i j}^{\kappa, p}$ given in subsections 5.4 and 5.5 to calculate $m_{i j}^{x, p}=\left.m_{i j}^{x}\right|_{\beta=0}$, we can show that the expression for the optimal tax rate simplifies to

$$
\tau(t)=\frac{\beta}{\alpha}+\frac{-\xi_{1}^{p}}{\phi}\left(k^{p}(t)-\tilde{k}^{p}\right)
$$

where

$$
\phi=\left(\frac{\alpha}{\beta}\right) B^{\frac{\alpha+\sigma}{(1-\alpha)(\sigma+\theta)}}\left(\frac{(1-\alpha) B}{\mu(1+\sigma)}\right)^{\frac{1}{\sigma+\theta}}\left(\frac{\alpha}{\rho}\right)^{\frac{\alpha(1+\sigma)}{(1-\alpha)(\sigma+\theta)}}>0
$$




\section{Additional Appendix (not for publication)}

\subsection{Control-State Space Analysis in the $(k, c / k)$ plane - the derivation of (61) and (62)}

In order to derive a system of differential equations in $k$ and $c / k$ we first introduce the definition $\eta \equiv c / k$. Substitution of $c=\eta k$ into (27)-(30) yields the following system:

$$
\begin{gathered}
\dot{k}=B k^{\alpha} l^{1-\alpha}-\eta k, \\
\eta^{-\theta} k^{-\theta}-\lambda=0, \\
-\mu(1+\sigma) l^{\sigma}+\lambda(1-\alpha) B(k / l)^{\alpha}=0, \\
\dot{\lambda} / \lambda=-\left[\alpha B(k / l)^{-(1-\alpha)}+\beta \eta-\rho\right] .
\end{gathered}
$$

Solving the necessary optimality conditions (70) and (71) for $l$ and $\lambda$ as functions of $k$ and $\eta$, we obtain

$$
\begin{gathered}
l=\left(\frac{(1-\alpha) B}{\mu(1+\sigma)}\right)^{\frac{1}{\alpha+\sigma}} k^{\frac{\alpha-\theta}{\alpha+\sigma}} \eta^{-\frac{\theta}{\alpha+\sigma}} \\
\lambda=\eta^{-\theta} k^{-\theta}
\end{gathered}
$$

Differentiating $\lambda=\eta^{-\theta} k^{-\theta}$ with respect to time $t$ we obtain

$$
\dot{\lambda} / \lambda=-\theta(\dot{\eta} / \eta)-\theta(\dot{k} / k)
$$

Substitution of (73) into (69) yields the differential equation

$$
\dot{k}=B\left(\frac{(1-\alpha) B}{\mu(1+\sigma)}\right)^{\frac{1-\alpha}{\alpha+\sigma}} k^{\frac{\alpha(1+\sigma)-\theta(1-\alpha)}{\alpha+\sigma}} \eta^{-\frac{(1-\alpha) \theta}{\alpha+\sigma}}-\eta k .
$$

that equals the differential equation (61).

Substituting (73) and (75) into (72) yields

$$
\dot{\eta}=\frac{\eta}{\theta}\left[\alpha B\left(\frac{(1-\alpha) B}{\mu(1+\sigma)}\right)^{\frac{1-\alpha}{\alpha+\sigma}} k^{-\frac{(1-\alpha)(\sigma+\theta)}{\alpha+\sigma}} \eta^{-\frac{(1-\alpha) \theta}{\alpha+\sigma}}+\beta \eta-\rho-\theta \frac{\dot{k}}{k}\right] .
$$

Using (76) to calculate $\dot{k} / k$ and substituting the resulting expression into (77), we finally obtain the differential equation (62):

$$
\dot{\eta}=\frac{\eta}{\theta}\left[(\alpha-\theta) B\left(\frac{(1-\alpha) B}{\mu(1+\sigma)}\right)^{\frac{1-\alpha}{\alpha+\sigma}} k^{-\frac{(1-\alpha)(\sigma+\theta)}{\alpha+\sigma}} \eta^{-\frac{(1-\alpha) \theta}{\alpha+\sigma}}+(\beta+\theta) \eta-\rho\right] .
$$




\subsection{Control-State Space Analysis in the $(k, k / l)$ plane - the derivation of (63) and (64)}

Letting $\kappa=k / l$, substitution of $l=k / \kappa$ into (27)-(30) yields the following system:

$$
\begin{gathered}
\dot{k}=B k \kappa^{-(1-\alpha)}-c, \\
c^{-\theta}-\lambda=0, \\
-\mu(1+\sigma)(k / \kappa)^{\sigma}+\lambda(1-\alpha) B \kappa^{\alpha}=0, \\
\dot{\lambda} / \lambda=-\left[\alpha B \kappa^{-(1-\alpha)}+\beta(c / k)-\rho\right] .
\end{gathered}
$$

Solving the necessary optimality conditions (79) and (80) for $c$ and $\lambda$ as functions of $k$ and $\kappa$ we obtain:

$$
\begin{gathered}
c=\left(\frac{(1-\alpha) B}{\mu(1+\sigma)}\right)^{\frac{1}{\theta}} k^{-\frac{\sigma}{\theta}} \kappa^{\frac{\alpha+\sigma}{\theta}}, \\
\lambda=\left(\frac{(1-\alpha) B}{\mu(1+\sigma)}\right)^{-1} k^{\sigma} \kappa^{-(\alpha+\sigma)} .
\end{gathered}
$$

Substitution of (82) into (78) yields

$$
\dot{k}=B k \kappa^{-(1-\alpha)}-\left(\frac{(1-\alpha) B}{\mu(1+\sigma)}\right)^{\frac{1}{\theta}} k^{-\frac{\sigma}{\theta}} \kappa^{\frac{\alpha+\sigma}{\theta}} .
$$

that equals the differential equation (63).

Differentiating (83) with respect to time $t$ we obtain

$$
\dot{\lambda} / \lambda=\sigma(\dot{k} / k)-(\alpha+\sigma)(\dot{\kappa} / \kappa) .
$$

Substitution of (82) and (85) into (81) yields

$$
\frac{\dot{\kappa}}{\kappa}=\frac{1}{\alpha+\sigma}\left[\alpha B \kappa^{-(1-\alpha)}+\beta\left(\frac{(1-\alpha) B}{\mu(1+\sigma)}\right)^{\frac{1}{\theta}} k^{-\frac{\sigma+\theta}{\theta}} \kappa^{\frac{\alpha+\sigma}{\theta}}+\sigma \frac{\dot{k}}{k}-\rho\right] .
$$

Using (84) to calculate $\dot{k} / k$ and substituting the resulting expression into (86), we finally obtain the differential equation (64).

$$
\dot{\kappa}=\frac{\kappa}{\alpha+\sigma}\left[(\alpha+\sigma) B \kappa^{-(1-\alpha)}+(\beta-\sigma)\left(\frac{(1-\alpha) B}{\mu(1+\sigma)}\right)^{\frac{1}{\theta}} k^{-\frac{\sigma+\theta}{\theta}} \kappa^{\frac{\alpha+\sigma}{\theta}}-\rho\right] .
$$




\section{References}

[1] Abel, A. B., 2005, Optimal Taxation When Consumers Have Endogenous Benchmark Levels of Consumption, Review of Economic Studies, 72(1), 21-42.

[2] Boskin, M. J. and E. Sheshinski, 1978, Optimal Redistributive Taxation when Individual Welfare Depends on Relative Income, The Quarterly Journal of Economics 92, $589-601$.

[3] Corneo, C. and O. Jeanne, 1997, On Relative Wealth Effects and the Optimality of Growth, Economics Letters 54 (1), pp. 87-92.

[4] Corneo, G. and O. Jeanne, 2001a, On Relative Wealth Effects and Long-Run Growth, Research in Economics 55, 349-358.

[5] Corneo, G., and O. Jeanne, 2001b, Status, the Distribution of Wealth, and Growth, Scandinavian Journal of Economics 103(2), 283-293.

[6] Dupor, B. and W.F. Liu, 2003, Jealousy and Equilibrium Overconsumption, American Economic Review 93(1), 423-428.

[7] Fisher, W. H., 2004, Status Preference, Wealth, and Dynamics in the Open Economy, German Economic Review 5(3), 335-355.

[8] Fisher, W. H. and F. X. Hof, 2000a, Relative Consumption, Economic Growth, and Taxation, Journal of Economics 72, 241-62.

[9] Fisher, W. H. and F. X. Hof, 2000b, Relative Consumption and Endogenous Labor Supply in the Ramsey Model: Do Status Conscious People Work Too Much?, Economics Series of the Institute for Advanced Studies No. 85, Vienna.

[10] Fisher, W. H. and F. X. Hof, 2005, Status Seeking in the Small Open Economy, Journal of Macroeconomics 27(2), 209-232.

[11] Frank, R.H., 1985, The Demand for Unobservable and Other Nonpositional Goods, The American Economic Review, March, 101-116.

[12] Futagami, Koichi, and Akihisa Shibata, 1998, Keeping one step ahead of the Joneses: status, the distribution of wealth, and long run growth, Journal of Economic Behavior and Organization (36) 1, pp. 93-111. 
[13] Gali, J., 1994, Keeping Up with the Joneses: Consumption Externalities, Portfolio Choice, and Asset Prices, Journal of Money, Credit, and Banking, vol. 26, no. 1, February, 1-8.

[14] Grossmann, V., 1998, Are Status Concerns Harmful for Growth?, FinanzArchiv 55 (3), 357-73.

[15] Harbaugh, R., 1996, Falling behind the Joneses: relative consumption and the growthsavings paradox, Economics Letters 53, 297-304.

[16] Hof, F.X., 2004, "Does the Quest for Status Give Rise to Excessive Work Effort and Excessive Consumption? - Relative Consumption Versus Relative Wealth", Vienna University of Technology, mimeo.

[17] Hof, F.X. and F. Wirl, 2003, Wealth Induced Multiple Equilibria in Small Open Economy Versions of the Ramsey Model, Vienna University of Technology, mimeo.

[18] Liu, W.F. and S.J. Turnovsky, 2005, Consumption Externalities, Production Externalities, and the Accumulation of Capital, Journal of Public Economics 89, 1097-1129.

[19] Ljungqvist, L. and H. Uhlig, 2000, Tax Policy and Aggregate Demand Management Under Catching Up with the Joneses, The American Economic Review 90, 356-66.

[20] Persson, M., 1995, Why are Taxes so High in Egalitarian Societies?, Scandinavian Journal of Economics 97(4), 569-580.

[21] Rauscher, M., 1997, Conspicuous Consumption, Economic Growth, and Taxation, Journal of Economics, Vol. 66, No. 1, 35-42.

[22] Turnovsky, S.J., 1995, Methods of Macroeconomic Dynamics, The MIT Press.

[23] Van Long, N. and K. Shimomura, 2004a, Relative Wealth, Status-Seeking, and Catching-Up, Journal of Economic Behavior \& Organization 53, 529-542.

[24] Van Long, N. and K. Shimomura, 2004b, Relative Wealth, Catching-up, and Economic Growth, in S. Dowrick, R. Pitchford, and S.J. Turnovsky, eds., Economic Growth and Macroeconomic Dynamics: Recent Developments in Economic Theory, New York: Cambridge University Press, 18-45. 
Figure 1: Phase Diagram in $(k, c)$

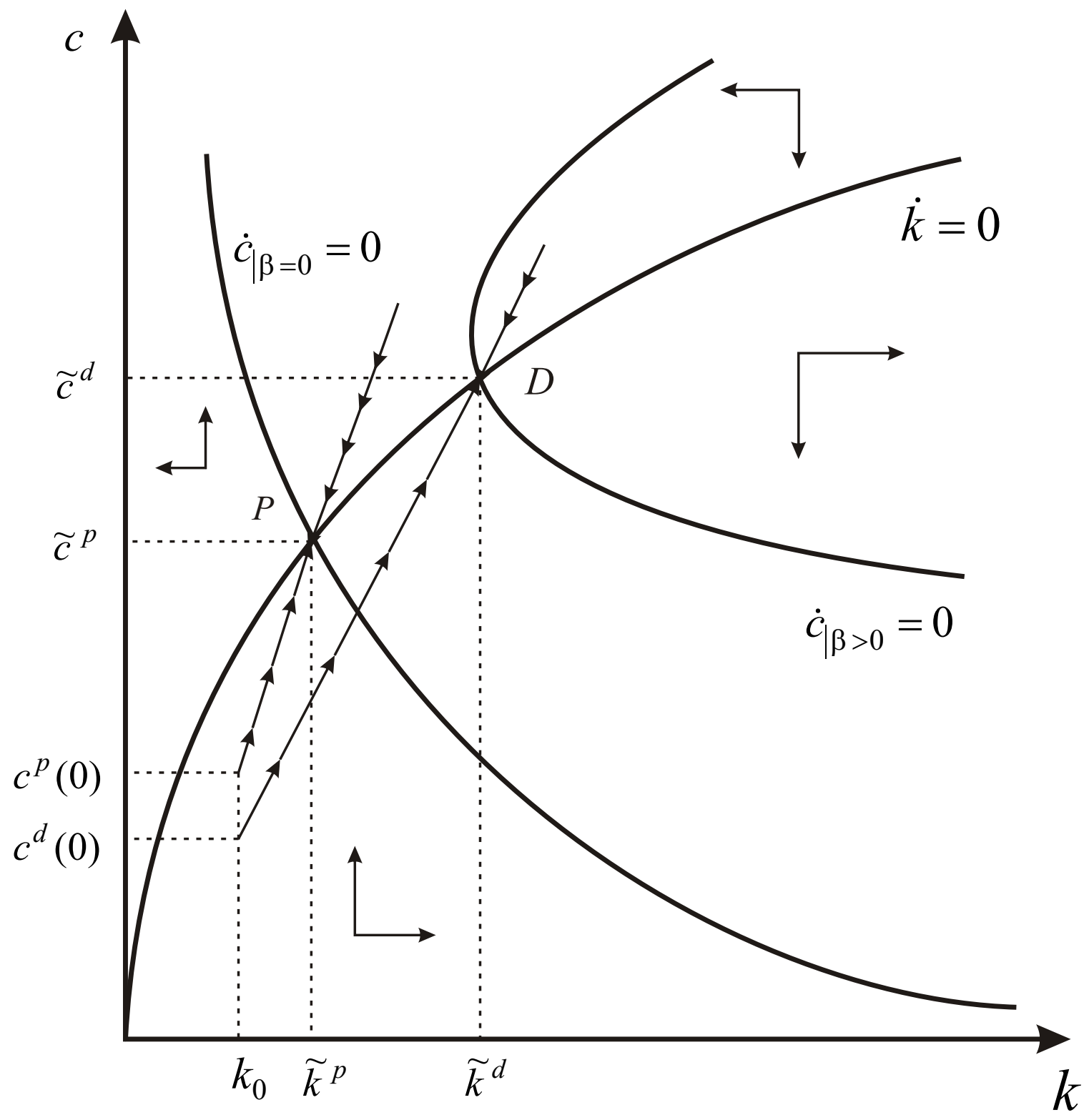


Figure 2a: Phase Diagram in $(k, l), \alpha=\theta<1$

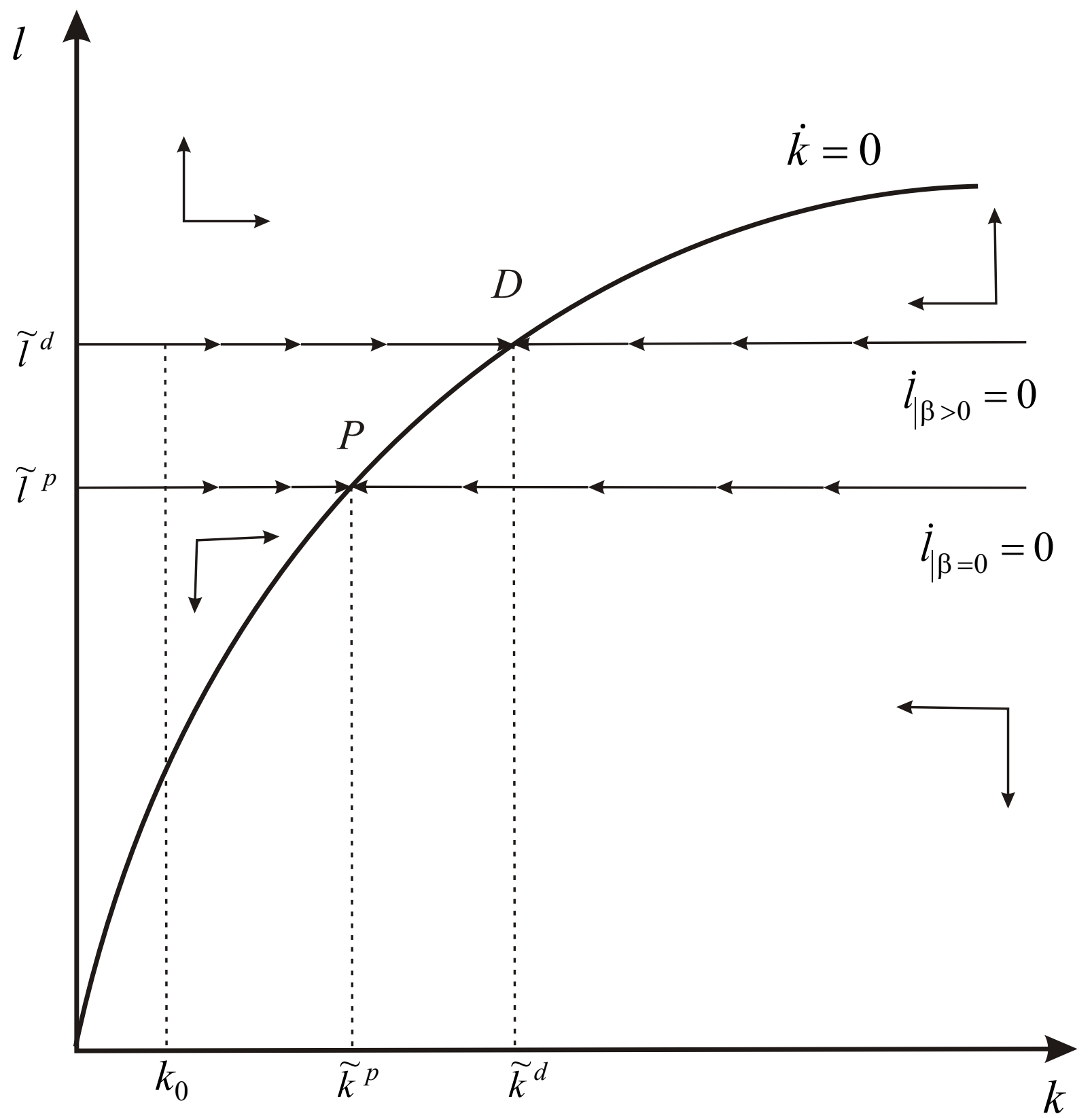


Figure 2b: Phase Diagram in $(k, l), \alpha<\theta=1$

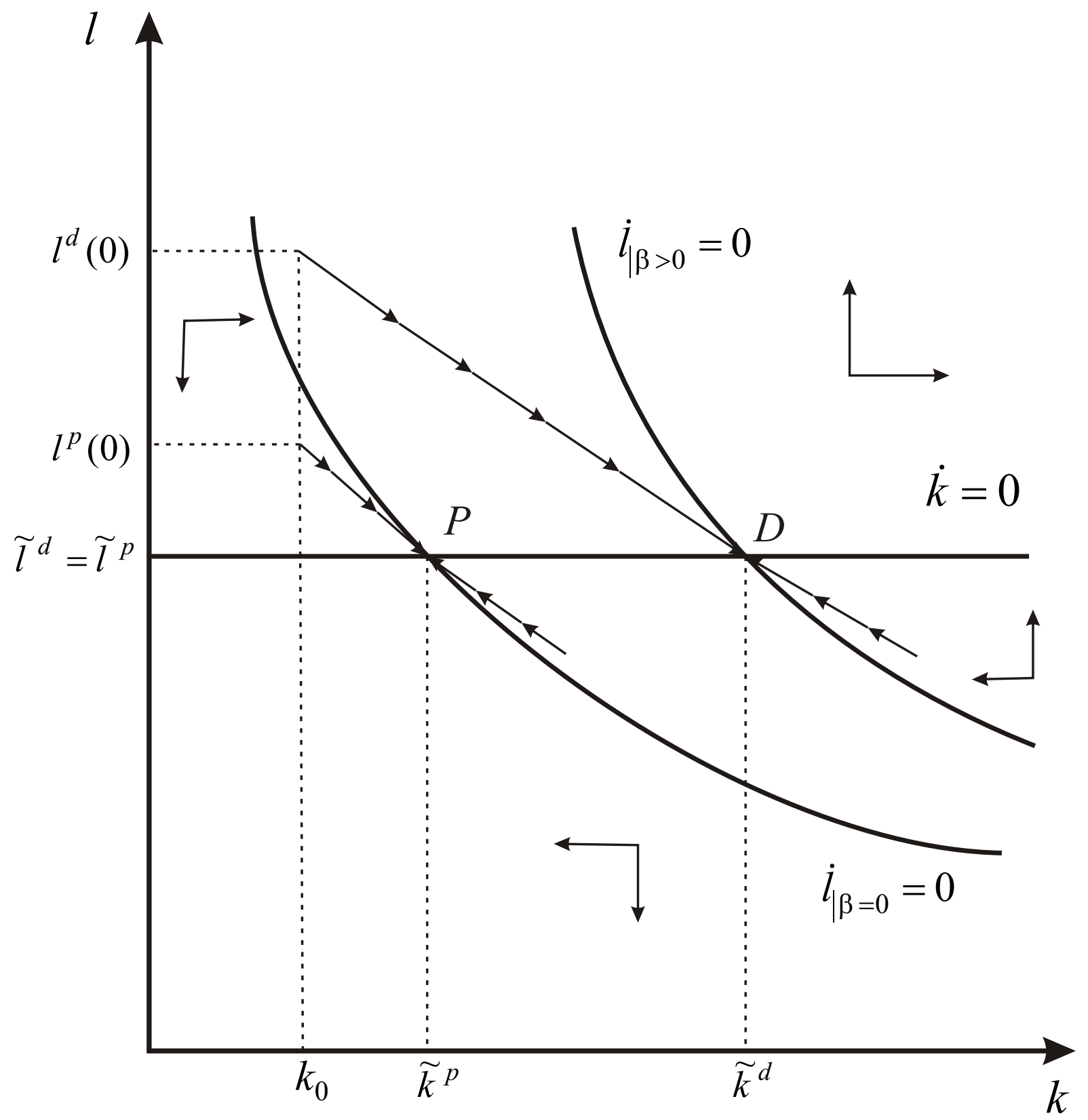


Figure 3a: The Paths of Decentralized and Planner's Employment for $\theta=0.25$

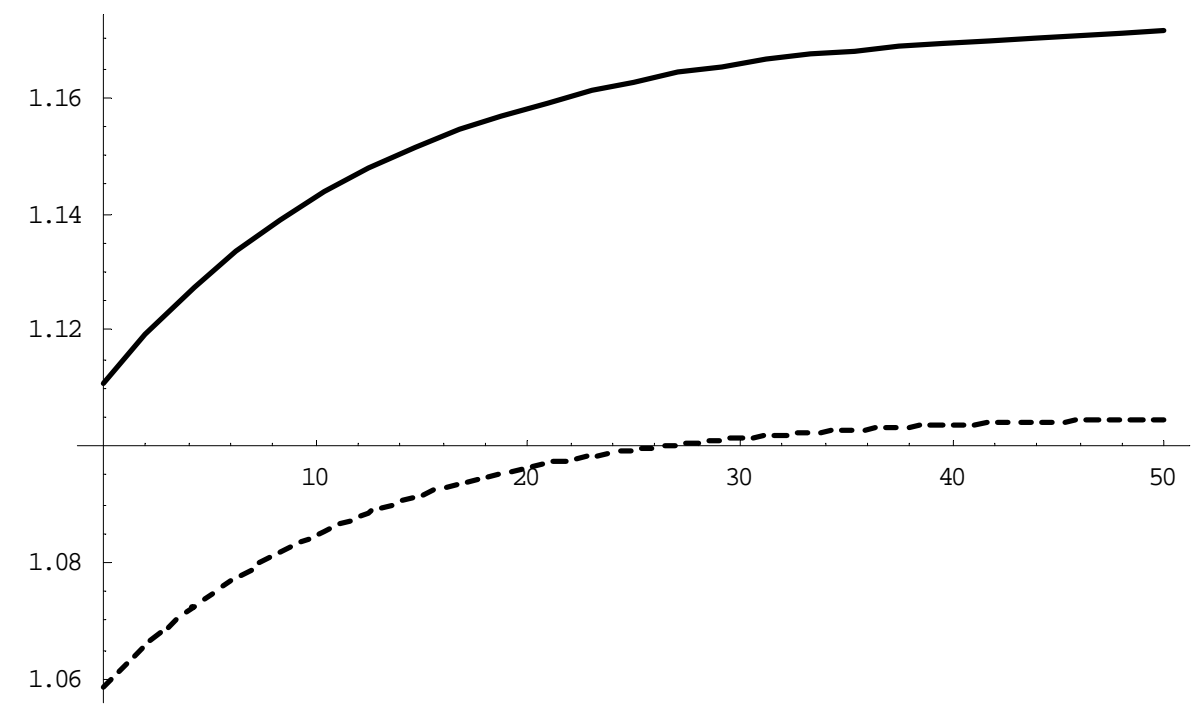

Figure 3b: The Paths of Decentralized and Planner's Employment for $\theta=2.0$

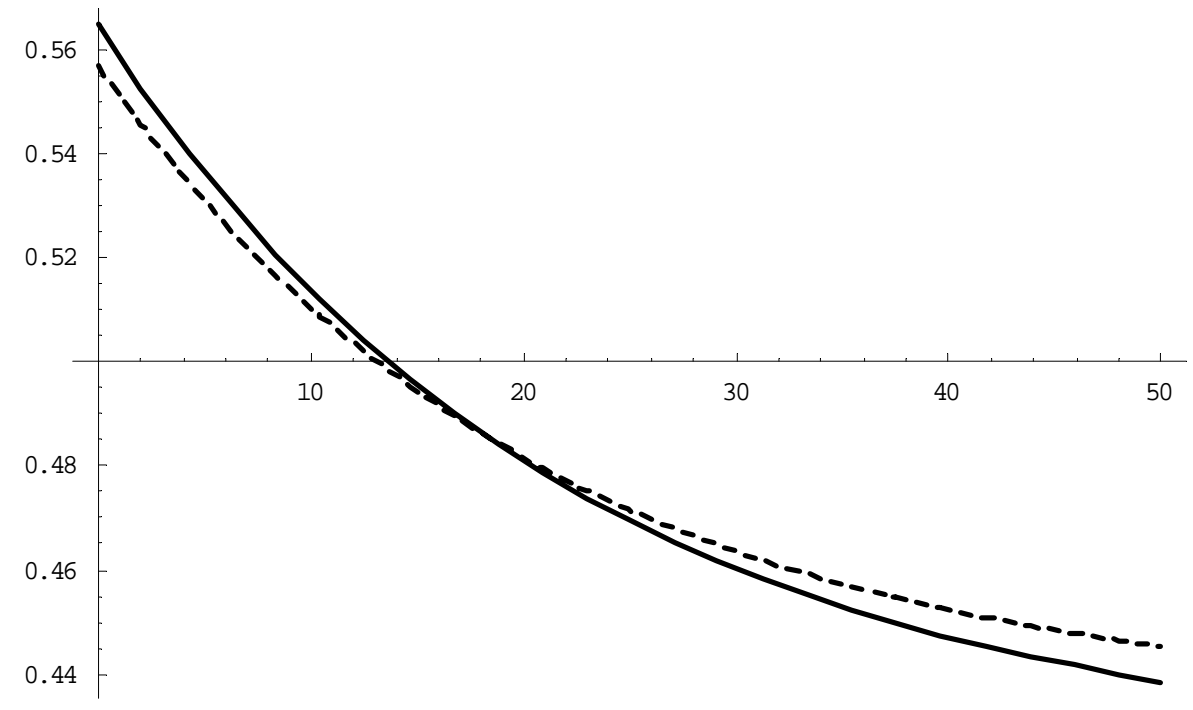


Figure 4: The Paths of Decentralized and Planner's Consumption for $\theta=0.25$

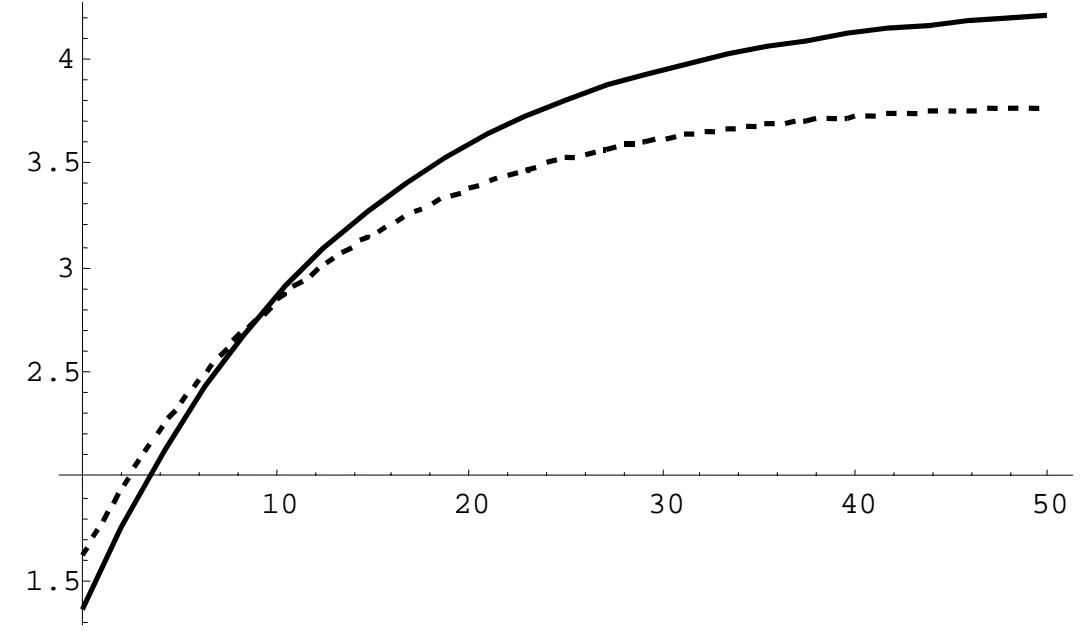

